TRANSACTIONS OF THE

AMERICAN MATHEMATICAL SOCIETY

Volume 352, Number 6, Pages 2801-2823

S 0002-9947(00)02521-6

Article electronically published on February 14, 2000

\title{
GEOMETRY OF BANACH SPACES HAVING SHRINKING APPROXIMATIONS OF THE IDENTITY
}

EVE OJA

\begin{abstract}
Let $a, c \geq 0$ and let $B$ be a compact set of scalars. We introduce property $M^{*}(a, B, c)$ of Banach spaces $X$ by the requirement that

$\limsup \left\|a x_{\nu}^{*}+b x^{*}+c y^{*}\right\| \leq \limsup \left\|x_{\nu}^{*}\right\| \quad \forall b \in B$

whenever $\left(x_{\nu}^{*}\right)$ is a bounded net converging weak ${ }^{*}$ to $x^{*}$ in $X^{*}$ and $\left\|y^{*}\right\| \leq$ $\left\|x^{*}\right\|$. Using $M^{*}(a, B, c)$ with $\max |B|+c>1$, we characterize the existence of certain shrinking approximations of the identity (in particular, those related to $M-, u$-, and $h$-ideals of compact or approximable operators). We also show that the existence of these approximations of the identity is separably determined.
\end{abstract}

\section{INTRODUCTION}

Let $X$ be a Banach space (over $\mathbb{K}=\mathbb{R}$ or $\mathbb{C}$ ) and let $I_{X}$ (or simply $I$ ) denote the identity operator on $X$. A net $\left(K_{\alpha}\right)$ of compact operators on $X$ is called a compact approximation of the identity $(C A I)$ provided $K_{\alpha} \longrightarrow I_{X}$ strongly (i.e. $K_{\alpha} x \longrightarrow x$ for any $x \in X)$. In particular, if $K_{\alpha}$ are finite rank operators, then $\left(K_{\alpha}\right)$ is called an approximation of the identity $(A I)$. If moreover, $K_{\alpha}^{*} \longrightarrow I_{X^{*}}$ strongly, then $\left(K_{\alpha}\right)$ is called shrinking (this notion can just be regarded as a generalization of shrinking bases). If there is an $\mathrm{AI}$ (resp. CAI) $\left(K_{\alpha}\right)$ with sup $\left\|K_{\alpha}\right\| \leq 1$, then $X$ is said to have the metric approximation property ( $M A P$ ) (resp. the metric compact approximation property $(M C A P))$. In this case, we shall say that $\left(K_{\alpha}\right)$ is a metric $A I(M A I)$ (resp. metric $C A I(M C A I))$. It is known that the MCAP does not imply the MAP [44]. In 1971, Johnson, Rosenthal, and Zippin [25] investigated under which conditions a Banach space with a basis has a shrinking basis. In [25] and later in [24] and [10, other cases of lifting an AI from a Banach space $X$ to $X^{*}$ were also considered. Johnson (see [24, Theorem 4 and its proof]) proved that if $X$ has an MAI for every equivalent norm, then $X$ has a shrinking MAI. In 1988, Godefroy and Saphar 17] demonstrated how the geometric structure of Banach spaces permits to lift metric approximation properties from spaces to their duals. They showed among others that if $X$ has an MAI (resp. MCAI) and $X^{*}$ contains no proper norming subspace, then $X$ has a shrinking MAI (resp. MCAI).

Received by the editors March 26, 1998.

2000 Mathematics Subject Classification. Primary 46B20, 46B28, 47L05.

This work was completed during a visit of the author to Freie Universität Berlin in 1997, supported by a grant from the Deutscher Akademischer Austauschdienst. The research was also partially supported by Estonian Science Foundation Grants 1820 and 3055.

The author is grateful to D. Werner for his hospitality and useful conversations. 
In the present paper, we shall be concerned with special cases of lifting the $\mathrm{M}(\mathrm{C}) \mathrm{AP}$ from $X$ to $X^{*}$ : we shall study the geometric structure of Banach spaces with the $\mathrm{M}(\mathrm{C}) \mathrm{AP}$ which guarantees the existence of shrinking (compact) approximations of the identity having certain important properties. The starting point of our investigations is the following fundamental result of Kalton and Werner [28. Theorem 2.7].

Theorem (Kalton-Werner). A separable Banach space $X$ has a shrinking MCAI $\left(K_{n}\right)_{n=1}^{\infty}$ satisfying $\lim \sup \left\|I_{X}-2 K_{n}\right\| \leq 1$ (equivalently, $\lim \left\|I_{X}-2 K_{n}\right\|=1$ ) whenever $X$ has the $M C A P$ and property $\left(M^{*}\right)$, i.e.

$$
\underset{\nu}{\limsup }\left\|x^{*}+x_{\nu}^{*}\right\|=\limsup _{\nu}\left\|y^{*}+x_{\nu}^{*}\right\|,
$$

for every $x^{*}, y^{*} \in X^{*}$ with $\left\|x^{*}\right\|=\left\|y^{*}\right\|$ and every bounded weak* null net $\left(x_{\nu}^{*}\right)$ in $X^{*}$.

(In [28, the sequential version of $\left(M^{*}\right)$ is used; both versions are equivalent whenever $X$ is separable.)

Some insight was then given into the Kalton-Werner theorem by Lima 31 who proved that the existence of the $\left(K_{n}\right)_{n=1}^{\infty}$ with the above properties in a separable Banach space $X$ with the MCAP is in fact equivalent to its property $\left(w M^{*}\right)$, i.e.

$$
\limsup _{\nu}\left\|2 x^{*}-x_{\nu}^{*}\right\|=\limsup _{\nu}\left\|x_{\nu}^{*}\right\|
$$

whenever $\left(x_{\nu}^{*}\right)$ is a bounded net converging weak ${ }^{*}$ to $x^{*}$ in $X^{*}$. Since $\left(w M^{*}\right)$ is (seemingly) weaker than $\left(M^{*}\right)$, Lima's argument actually gives a new proof of the Kalton-Werner theorem which is somewhat shorter and simpler than the original one. Both these proofs rely on the separability of $X$. However, with a different argument, Lima [31] also established similar results for reflexive spaces.

The following theorem is the main result of this paper (see Theorem 3.5 in Section 3$)$. We denote by $\mathcal{K}(X)$ the Banach space of compact operators on $X$ and by $B_{\mathcal{K}(X)}$, its closed unit ball.

Theorem. Let $a, c \geq 0$ and let $B \subset \mathbb{K}$ be a compact set. If $\max |B|+c>1$, then the following assertions are equivalent for a Banach space $X$.

$1^{\circ}$. For every $S \in B_{\mathcal{K}(X)}$, there exists a shrinking MAI (resp. MCAI) $\left(K_{\alpha}\right)$ such that

$$
\limsup _{\alpha}\left\|a I_{X}+b K_{\alpha}+c S\right\| \leq 1 \quad \forall b \in B .
$$

$2^{\circ} . X$ has the MAP (resp. MCAP) and

$$
\underset{\nu}{\limsup }\left\|a x_{\nu}^{*}+b x^{*}+c y^{*}\right\| \leq \underset{\nu}{\limsup }\left\|x_{\nu}^{*}\right\| \quad \forall b \in B
$$

whenever $\left(x_{\nu}^{*}\right)$ is a bounded net converging weak $k^{*}$ to $x^{*}$ in $X^{*}$ and $\left\|y^{*}\right\| \leq$ $\left\|x^{*}\right\|$.

$3^{\circ}$. X has the MAP (resp. MCAP) and, for every separable closed subspace $Y$ of $X$ having the MAP (resp. MCAP), there exists a shrinking MAI (resp. $M C A I)\left(K_{n}\right)_{n=1}^{\infty}$ such that

$$
\limsup _{n}\left\|a I_{Y}+b K_{n}+c S\right\| \leq 1 \quad \forall b \in B, \quad \forall S \in B_{\mathcal{K}(Y)} .
$$


The Theorem characterizes intrinsically a large class of shrinking (C)AI, including e.g. those (related to $u$-ideals) for which limsup $\left\|I_{X}-2 K_{\alpha}\right\| \leq 1$, studied by Casazza, Emmanuele, Godefroy, Kalton, Li, Lima, Rao, Saphar, and Werner (4], [9], [12], [13], [14], [15], [16], [28], [31]), those (related to $h$-ideals) for which lim sup $\left\|I_{X}-(1+\lambda) K_{\alpha}\right\| \leq 1$ whenever $\lambda \in \mathbb{C}$ satisfies $|\lambda|=1$ (or, more generally, [16], lim sup $\left\|I_{X}-\lambda K_{\alpha}\right\| \leq \mu$ for every $\lambda$ from a given compact set $\Lambda \subset \mathbb{K}$ ), studied by Godefroy, Kalton, Li, and Saphar ([12], [14], 15], 16], [29]), and those related to $M$-ideals of compact operators (cf. [20, p. 299], [26], [27, [34], [36]). The Theorem also shows that the existence of these approximations of the identity is separably determined. This fact seems to be important since special classes of approximations of the identity have mainly been studied in separable Banach spaces using results limited to the separable case (cf., e.g., 4, 16], and their bibliographies).

The structural property of Banach spaces described in condition $2^{\circ}$ of the Theorem will be called property $M^{*}(a, B, c)$. Property $\left(M^{*}\right)$ is the same as $M^{*}(1,\{-1\}, 1)$ and $\left(w M^{*}\right)$ is $M^{*}(1,\{-2\}, 0)$. In Section 1, applying, among others, techniques from [4], [16], 27], we make a preliminary study of property $M^{*}(a, B, c)$. We show, for example, that any separable Banach space with separable dual can be equivalently renormed to have property $M^{*}(1,\{b:|b+1| \leq r\}, 0)$, where $0 \leq r<1$. On the other hand, we show that any MCAI in a Banach space $X$ is shrinking whenever $X$ satisfies $M^{*}(a, B, c)$ with $\max |B|+c>1$. The (easy) implication $1^{\circ} \Longrightarrow 2^{\circ}$ of the Theorem is also proved in Section 1.

The implication $2^{\circ} \Longrightarrow 3^{\circ}$ is established in Section 2. Its proof relies on the Simons sup-lim sup theorem. For separable $X$, this already completes the proof of the Theorem. The separable version of the Theorem contains, in particular, Lima's and the Kalton-Werner theorems providing their new and simpler proof. In the complex case, it also strengthens the Kalton-Werner theorem, showing that then $\left(K_{n}\right)_{n=1}^{\infty}$ actually satisfies $\lim \left\|I_{X}-(1+\lambda) K_{n}\right\|=1$ whenever $|\lambda|=1$. The Kalton-Werner theorem and Theorem 2.4 in [27] by Kalton immediately imply that a separable Banach space $X$ has the $M C A P$ and $\left(M^{*}\right)$ if and only if there exists a shrinking $M C A I\left(K_{n}\right)_{n=1}^{\infty}$ such that limsup $\left\|I_{X}-K_{n}+S\right\| \leq 1$ for every $S \in B_{\mathcal{K}(X)}$. (This is a basic result of the theory of $M$-ideals of compact operators.) The separable version of the Theorem contains this result providing its direct and much easier proof.

In Section 3, using an inductive technical construction, we show that $3^{\circ} \Longrightarrow 1^{\circ}$. This completes the proof of the Theorem extending, in particular, Lima's and the Kalton-Werner theorems - together with their complex versions - to arbitrary (nonseparable) Banach spaces.

In Section 4, results of Sections 1-3 are applied to study special classes of ideals of compact and approximable operators. In particular, this provides an alternative unified and easier approach to the theories of $M_{-}, u-$, and $h$-ideals of compact operators (cf. [20, Chapter VI] together with [28], and [4], [16], [31]).

Let us now fix some more notation. In a Banach (or normed linear) space $X$, we denote the unit sphere by $S_{X}$ and the closed unit ball by $B_{X}$. For a set $A \subset X$, its norm closure is denoted by $\bar{A}$, its linear span by span $A$, and its convex hull by $\operatorname{conv} A$. We denote the set of all weak* strongly exposed points of $B_{X^{*}}$ by $w^{*}$-sexp $B_{X^{*}}$. For a set $A \subset X^{*}$, its weak* closure is denoted by $\bar{A}^{w^{*}}$. Further, let $\pi_{X}$ be the canonical projection of $X^{* * *}$ onto $X^{*}$ (that is, $\pi_{X}=j_{X^{*}}\left(j_{X}\right)^{*}$ where $j_{X}: X \longrightarrow X^{* *}$ is the canonical embedding). And, finally, we denote by $\mathcal{L}(X)$ the 
Banach space of all bounded linear operators on $X$ and by $\mathcal{A}(X)$ its subspace of approximable operators (i.e., norm limits of finite rank operators).

\section{General facts about property $M^{*}(a, B, c)$}

Throughout this paper, $B \subset \mathbb{K}$ will be a compact set and $a, c \geq 0$. We shall say that a Banach space $X$ has property $M^{*}(a, B, c)$ if

$$
\underset{\nu}{\limsup }\left\|a x_{\nu}^{*}+b x^{*}+c y^{*}\right\| \leq \limsup _{\nu}\left\|x_{\nu}^{*}\right\| \quad \forall b \in B
$$

whenever $x^{*}, y^{*} \in X^{*}$ satisfy $\left\|y^{*}\right\| \leq\left\|x^{*}\right\|$, and $\left(x_{\nu}^{*}\right)$ is a bounded net converging weak ${ }^{*}$ to $x^{*}$ in $X^{*}$.

It can easily be seen that $M^{*}(1,\{-1\}, 1)$ is precisely property $\left(M^{*}\right)$ introduced by Kalton [27] (see also [26]) and $M^{*}(1,\{-2\}, 0)$ is property $\left(w M^{*}\right)$ introduced by Lima 31]. It will turn out that $M^{*}(1,\{b:|b+1|=1\}, 0)$ is the natural complex version of $\left(w M^{*}\right)$. This property easily follows from $M^{*}(1,\{-1\}, 1)=\left(M^{*}\right)$. More generally, it is straightforward to verify that $\left(M^{*}\right)$ implies $M^{*}(1,\{b:|b+1| \leq 1-c\}, c)$ for any $c \in[0,1]$.

It is well known (and easy to check) that, for example, the spaces $\ell_{p}, 1<p<\infty$, satisfy property $\left(M^{*}\right)$ but the Lorentz sequence spaces $d(w, p)$ do not. On the other hand, it is straightforward to verify that $d(w, p), 1<p<\infty$, satisfies property $M^{*}(a, B, c)$ for any fixed $a, c>0$ such that $a^{p}+c^{p} \leq 1$ and $B=\{b:|b+a| \leq$ $\left.\left(1-a^{p}\right)^{1 / p}-c\right\}$. From Lemma 1.1 below (cf. also [31, Theorem 4.2]), it will be clear that $d(w, p)$ and, more generally, Banach spaces with a shrinking 1-unconditional basis enjoy property $\left(w M^{*}\right)$ and, in the case of complex scalars, its complex version.

A separable Banach space $X$ has $M^{*}(a, B, c)$ if and only if it has the sequential version of $M^{*}(a, B, c)$. This can easily be checked using the fact that closed balls in $X^{*}$ are weak* metrizable.

Property $M^{*}(a, B, c)$ is trivially inherited by quotient spaces. It is also inherited by subspaces. In fact, suppose that $X$ has property $M^{*}(a, B, c)$. If its subspace $Y$ does not have $M^{*}(a, B, c)$, then

$$
\underset{\nu}{\limsup }\left\|a y_{\nu}^{*}+b y^{*}+c w^{*}\right\|>\underset{\nu}{\limsup }\left\|y_{\nu}^{*}\right\|
$$

for some $b \in B, y^{*}, w^{*} \in Y^{*}$ with $\left\|w^{*}\right\| \leq\left\|y^{*}\right\|$, and a bounded net $\left(y_{\nu}^{*}\right)$ converging weak* to $y^{*}$ in $Y^{*}$. By passing to a subnet, we may assume that

$$
\lim \left\|a y_{\nu}^{*}+b y^{*}+c w^{*}\right\|>\lim \left\|y_{\nu}^{*}\right\| .
$$

Let $x_{\nu}^{*}$ and $z^{*}$ be norm-preserving extensions of $y_{\nu}^{*}$ and $w^{*}$ to $X$. There exists a subnet $\left(x_{\nu(\mu)}^{*}\right)$ of $\left(x_{\nu}^{*}\right)$ converging weak ${ }^{*}$ to some $x^{*}$ in $X^{*}$. Clearly, $x^{*}$ extends $y^{*}$. Using property $M^{*}(a, B, c)$ of $X$, we get

$$
\begin{aligned}
\lim _{\nu}\left\|a y_{\nu}^{*}+b y^{*}+c w^{*}\right\| & =\lim _{\mu}\left\|\left.\left(a x_{\nu(\mu)}^{*}+b x^{*}+c z^{*}\right)\right|_{Y}\right\| \\
& \leq \limsup _{\mu}\left\|x_{\nu(\mu)}^{*}\right\|=\lim _{\nu}\left\|y_{\nu}^{*}\right\| .
\end{aligned}
$$

The contradiction proves that $Y$ has property $M^{*}(a, B, c)$.

The following simple lemma (which will be needed in Section 3 below to prove the main result of the paper) shows that the existence of certain approximations of the identity implies $M^{*}(a, B, c)$. 
Lemma 1.1. A Banach space $X$ has property $M^{*}(a, B, c)$ whenever, for any rank one operator $S$ on $X$ with $\|S\| \leq 1$, there exists a shrinking $C A I\left(K_{\alpha}\right)$ such that

$$
\limsup _{\alpha}\left\|a I_{X}+b K_{\alpha}+c S\right\| \leq 1 \quad \forall b \in B .
$$

Proof. Let $\left(x_{\nu}^{*}\right)$ be a bounded net converging weak ${ }^{*}$ to $x^{*}$ in $X^{*}$. It is clearly enough to consider the case when $\left\|y^{*}\right\|<\left\|x^{*}\right\|$. Choose $x \in X$ so that $x^{*}(x)=1$ and $\left\|y^{*}\right\|<1 /\|x\|$. Denoting $S=y^{*} \otimes x$, we have $\|S\|<1$ and $y^{*}=S^{*} x^{*}$. Let $\left(K_{\alpha}\right)$ be given by assumption. Since $\left\|K^{*}\left(x^{*}-x_{\nu}^{*}\right)\right\| \longrightarrow 0$ if $K$ is compact, we get, for any fixed $\alpha$,

$$
\begin{aligned}
& \limsup \left\|a x_{\nu}^{*}+b x^{*}+c y^{*}\right\| \leq \lim \sup _{\nu}\left\|\left(a I_{X^{*}}+b K_{\alpha}^{*}+c S^{*}\right) x_{\nu}^{*}\right\|
\end{aligned}
$$

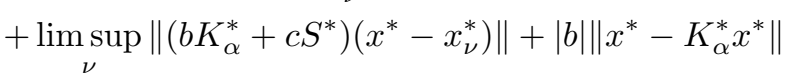

$$
\begin{aligned}
& \leq\left\|a I_{X}+b K_{\alpha}+c S\right\| \limsup _{\nu}\left\|x_{\nu}^{*}\right\|+|b|\left\|x^{*}-K_{\alpha}^{*} x^{*}\right\|,
\end{aligned}
$$

and the conclusion follows by taking $\lim \sup _{\alpha}$.

The idea of the proof of the next proposition comes from Theorem 2.9 of 16 that, in turn, follows from Propositions 1.2 and 1.3 below.

Proposition 1.2. Suppose $0 \leq r<1$ and denote $B=\{b:|b+1| \leq r\}$. Then any separable Banach space $X$ with separable dual can be equivalently renormed to have property $M^{*}(1, B, 0)$.

Proof. According to a theorem due to Zippin [45], $X$ is isomorphic to a subspace of a Banach space with a shrinking basis. Since property $M^{*}(a, B, c)$ is inherited by subspaces, we may assume that $X$ has a shrinking basis. Let $\left(P_{n}\right)_{n=1}^{\infty}$ be the associated partial sum operators. By the proof of [4, Lemma 3.4], $X$ can be equivalently renormed so that $\sup _{n}\left\|I+b P_{n}\right\| \leq 1$ for all $b \in B$. Thus the result is immediate from Lemma 1.1.

Remark. The above renorming is no longer possible for $r=1$, e.g., James space $J$ cannot be equivalently renormed to have property $M^{*}(1,\{-2\}, 0)=\left(w M^{*}\right)$. (In fact, it follows from [31, Proposition 4.1] and [16, Proposition 5.2 and Theorem 5.4] that $\left(w M^{*}\right)$ implies Pełczyński's property $(u)$. But then, if the space happens to be nonreflexive and to have a separable dual, it must contain an isomorphic copy of $c_{0}$.)

The proof of the following proposition is similar to the proof of the fact that $X$ is an $M$-ideal in its bidual whenever $X$ has $\left(M^{*}\right)$ (cf. [27] or [20, p. 298]) and will therefore be omitted. We shall use Proposition 1.3 in Section 4 below.

Proposition 1.3. If a Banach space $X$ has property $M^{*}(a, B, c)$, then the natural projection $\pi_{X}: X^{* * *} \longrightarrow X^{*}$ satisfies the inequality

$$
\left\|a x^{* * *}+b \pi_{X} x^{* * *}\right\|+c\left\|\pi_{X} x^{* * *}\right\| \leq\left\|x^{* * *}\right\| \quad \forall b \in B, \quad \forall x^{* * *} \in X^{* * *} .
$$

The following result is now immediate. It extends Theorem 2.9 of [16] to the case of complex scalars.

Corollary 1.4. Let $X$ be a nonreflexive separable Banach space for which $X^{*}$ is separable. If $0 \leq r<1$, then $X$ can be equivalently renormed so that $\| I_{X^{* * *}}-$ $\lambda \pi_{X} \|=1$ whenever $|\lambda-1| \leq r$ (i.e. $\{\lambda \in \mathbb{K}:|\lambda-1| \leq r\}$ belongs to the Godun set of $X$ ). 
We conclude this section by studying implications of property $M^{*}(a, B, c)$ with $\max |B|+c>1$ (satisfied, in particular, for $\left(M^{*}\right)$ and (the complex version of) $\left.\left(w M^{*}\right)\right)$. We shall see, in this case, that any $\mathrm{M}(\mathrm{C}) \mathrm{AI}$ will already be shrinking.

Let us recall that the characteristic $r(V)$ of a subspace $V$ of $X^{*}$ is defined by

$$
r(V)=\max \left\{r \geq 0: r B_{X^{*}} \subset{\overline{B_{V}}}^{w^{*}}\right\} .
$$

Obviously, $r(V) \leq 1$.

Proposition 1.5. If a Banach space $X$ satisfies property $M^{*}(a, B, c)$ with $\max |B|$ $+c>1$, then $r(V) \leq 1 /(\max |B|+c)<1$ for any proper closed subspace $V$ of $X^{*}$.

Proof. One can prove Proposition 1.5 by relying on Proposition 1.3 and some results from [16] (see Section 4 below, Theorem 4.1, proof of $1^{\circ \circ} \Longrightarrow 1^{\circ}$ ). However, we prefer to give a direct proof. Put $\rho=1 /(\max |B|+c)$. Since $r(V) \leq r(W)$ if $V \subset W$, it is enough to consider the case when $V=\operatorname{ker} x^{* *}$ where $x^{* *} \in S_{X^{* *}}$. We argue by contradiction. Suppose that $(\rho+\epsilon) B_{X^{*}} \subset{\overline{B_{V}}}^{w^{*}}$ for some $\epsilon>0$ and choose $x^{*} \in X^{*}$ with $\left\|x^{*}\right\|=\rho+\epsilon$ so that $\left|x^{* *}\left(x^{*}\right)\right|>\rho$. Then $B_{V}$ contains a net $\left(x_{\nu}^{*}\right)$ converging weak ${ }^{*}$ to $x^{*}$. Note that $x^{* *}\left(x_{\nu}^{*}\right)=0$ for all $\nu$. Let $|b|=\max |B|=b \operatorname{sgn} b$ for some $b \in B$. Since $\operatorname{sgn} b x_{\nu}^{*} \longrightarrow \operatorname{sgn} b x^{*}$ weak $^{*}$ and $\left\|x^{*}\right\|=\left\|\operatorname{sgn} b x^{*}\right\|$, we have

$$
\limsup _{\nu}\left\|a \operatorname{sgn} b x_{\nu}^{*}+|b| x^{*}+c x^{*}\right\| \leq 1 .
$$

But then

$$
1 \geq \underset{\nu}{\lim \sup }\left|x^{* *}\left(a \operatorname{sgn} b x_{\nu}^{*}+|b| x^{*}+c x^{*}\right)\right|=\frac{1}{\rho}\left|x^{* *}\left(x^{*}\right)\right|>1,
$$

a contradiction.

Remark. It is well known that $c_{0}$ has property $\left(M^{*}\right)\left(=M^{*}(1 ;\{-1\}, 1)\right)$. If $V=$ $\left\{\left(\alpha_{n}\right): \sum_{n=1}^{\infty} \alpha_{n}=0\right\} \subset \ell_{1}=c_{0}^{*}$, then $r(V)=1 / 2$.

Since property $M^{*}(a, B, c)$ is inherited by subspaces and quotient spaces, Propositions 1.2 and 1.5 immediately yield Corollary 2.10 of [16] asserting that if $\epsilon>0$, then any separable Banach space $X$ with separable dual can be equivalently renormed so that, for any subspace $Y$ of a quotient space of $X$, whenever $V$ is a proper closed subspace of $Y^{*}$, then $r(V) \leq \frac{1}{2}+\epsilon$. Let us recall that the question about the existence of such a renorming comes from the paper [7] by van Dulst and Singer (cf. also [11]).

Corollary 1.6. If a Banach space $X$ satisfies property $M^{*}(a, B, c)$ with $\max |B|+$ $c>1$, then $X^{*}$ contains no proper norming closed subspace, $X$ is an Asplund space, and

$$
X^{*}=\overline{\operatorname{span}}\left(w^{*}-\operatorname{sexp} B_{X^{*}}\right) .
$$

Proof. The first claim is immediate from Proposition 1.5. Since subspaces of $X$ inherit property $M^{*}(a, B, c)$, duals of its separable subspaces (they contain separable norming subspaces) contain no proper norming subspace and therefore they must be separable. This means that $X$ is an Asplund space. Finally, since $X$ is an Asplund space, $B_{X^{*}}=\overline{\operatorname{conv}} w^{*}\left(w^{*}-\operatorname{sexp} B_{X^{*}}\right)$ (cf., e.g., [38, p. 86]). Hence $\overline{\operatorname{span}}\left(w^{*}-\operatorname{sexp} B_{X^{*}}\right)$ is a norming subspace and therefore must be equal to $X^{*}$. 
Corollary 1.6 and a result of Godefroy and Saphar [17, p. 679 and p. 680] immediately yield that if a Banach space $X$ satisfying $M^{*}(a, B, c)$ with $\max |B|+c>1$ has an MAI (resp. MCAI), then $X$ has a shrinking MAI (resp. MCAI). However here the following refinement is possible.

Corollary 1.7. Let $X$ be a Banach space satisfying property $M^{*}(a, B, c)$ with $\max |B|+c>1$ and let $Y$ be a closed subspace of a quotient space of $X$. If $Y$ has an $M(C) A I\left(K_{\alpha}\right)$, then $\left(K_{\alpha}\right)$ is shrinking.

Proof. Let $K_{\alpha} y \longrightarrow y, y \in Y$, and $\sup \left\|K_{\alpha}\right\| \leq 1$. By the above, $Y^{*}=$ $\overline{\operatorname{span}}\left(w^{*}-\operatorname{sexp} B_{Y^{*}}\right)$, and therefore it suffices to show that $K_{\alpha}^{*} y^{*} \longrightarrow y^{*}$ whenever $y^{*} \in B_{Y^{*}}$ is strongly exposed by some $y$. But this is implied by $\left(K_{\alpha}^{*} y^{*}\right)(y) \longrightarrow$ $y^{*}(y)=1$ since $K_{\alpha}^{*} y^{*} \in B_{Y^{*}}$.

Corollary 1.7 will be further developed in Corollary 2.3 below.

Arguing as in [17 Corollary 4.4 and Proposition 4.3], we can derive from Proposition 1.5 the following refinement of Corollary 1.7 for basic sequences.

Corollary 1.8. Let $X$ be a Banach space satisfying property $M^{*}(a, B, c)$ with $\max |B|+c>1$ and let $Y$ be a closed subspace of a quotient space of $X$. Let $\left(e_{n}\right)$ be a basic sequence in $Y$. If the basis constant of $\left(e_{n}\right)$ is strictly less than $\max |B|+c$, then $\left(e_{n}\right)$ is shrinking.

\section{The IMPACt of PROPERTy $M^{*}(a, B, c)$ \\ ON SHRINKING APPROXIMATIONS OF THE IDENTITY}

The next lemma is fundamental for the results of the present paper. It shows that property $M^{*}(a, B, c)$ has a special impact on shrinking approximations of the identity.

Lemma 2.1. Let $X$ be a Banach space with property $M^{*}(a, B, c)$ and let $Y$ be a separable closed subspace of a quotient space of $X$. If $Y$ has a shrinking $C A I$ $\left(L_{n}\right)_{n=1}^{\infty}$, then there exist $K_{n} \in \operatorname{conv}\left\{L_{n}, L_{n+1}, \ldots\right\}, n \in \mathbb{N}$, so that

$$
\underset{n}{\limsup }\left\|a I_{Y}+b K_{n}+c S\right\| \leq 1 \quad \forall b \in B, \quad \forall S \in B_{\mathcal{K}(Y)} .
$$

Remark. It is known (this is an extension of classical results of Grothendieck) that whenever an Asplund space has a shrinking AI (resp. CAI), it also has a shrinking MAI (resp. MCAI) (cf. [17, Theorem 1.5], [5] or [6, p. 246] (for the case of AI)).

The proof of Lemma 2.1 relies on the following variant of the Simons sup-lim sup theorem. For the sake of completeness, we present here its direct easy proof (cf. [39, Lemma 2, Theorem 3]) which does not depend on an eigenvector argument (cf. [40]).

Theorem 2.2 (Simons). Let $\left(x_{n}\right)_{n=1}^{\infty}$ be a bounded sequence in a Banach space $X$. Let $G$ be a bounded subset of $X^{*}$ and let $F \subset G$ be such that, for all $x=$ $\sum_{n=1}^{\infty} \lambda_{n} x_{n}$ with $\lambda_{n} \geq 0$ and $\sum_{n=1}^{\infty} \lambda_{n}=1$, there exists $f \in F$ satisfying $\operatorname{Re} f(x)=$ $\sup _{g \in G} \operatorname{Re} g(x)$. Then

$$
\sigma_{F}:=\sup _{f \in F} \limsup _{n} \operatorname{Re} f\left(x_{n}\right)=\sup _{g \in G} \limsup _{n} \operatorname{Re} g\left(x_{n}\right)=: \sigma_{G} .
$$


Proof. Since $F \subset G$, we have $\sigma_{F} \leq \sigma_{G}$. Assume, on the contrary, that $\sigma_{F}<\sigma_{G}$. Then $\sigma_{F}<\limsup \operatorname{Re} g_{0}\left(x_{n}\right)$ for some $g_{0} \in G$. Hence, there are $\delta>0$ and a subsequence $\left(y_{n}\right)_{n=1}^{\infty}$ of $\left(x_{n}\right)_{n=1}^{\infty}$ so that

$$
\sigma_{F}+\delta<\inf _{n} \operatorname{Re} g_{0}\left(y_{n}\right) .
$$

Denote $\sigma(x)=\sup _{g \in G} \operatorname{Re} g(x), x \in X$, and

$$
C_{k}=\left\{\sum_{n=k}^{\infty} \lambda_{n} y_{n}: \lambda_{n} \geq 0, \sum_{n=k}^{\infty} \lambda_{n}=1\right\}, \quad k \in \mathbb{N} .
$$

Since $C_{k}$ is a bounded set,

$$
\inf _{z \in C_{k}} \sigma(x+z)>-\infty \quad \forall x \in X, \quad \forall k \in \mathbb{N} .
$$

Choose inductively $z_{1} \in C_{1}, z_{2} \in C_{2}, \ldots$ so that

$$
\sigma\left(2^{k} v_{k}+z_{k+1}\right) \leq \inf _{z \in C_{k+1}} \sigma\left(2^{k} v_{k}+z\right)+\frac{\delta}{2^{k+1}}, \quad k=0,1, \ldots,
$$

where $v_{0}=0$ and $v_{k}=\sum_{n=1}^{k} z_{n} / 2^{n}$. Then put $v=\sum_{n=1}^{\infty} z_{n} / 2^{n}$. Since $z_{k+1}=$ $2^{k+1} v_{k+1}-2^{k+1} v_{k}$ and $2^{k} v-2^{k} v_{k}=\sum_{n=k+1}^{\infty} 2^{k} z_{n} / 2^{n} \in C_{k+1}$, we have

$$
\sigma\left(2^{k+1} v_{k+1}-2^{k} v_{k}\right) \leq \sigma\left(2^{k} v\right)+\frac{\delta}{2^{k+1}}=2^{k} \sigma(v)+\frac{\delta}{2^{k+1}}, \quad k=0,1, \ldots .
$$

Since $v \in C_{1}$, there exists $f \in F$ satisfying $\operatorname{Re} f(v)=\sigma(v)$. From (2) (note that $\left.\sum_{k=0}^{m-1} 2^{k}=2^{m}-1\right)$ we immediately get that

$$
\begin{aligned}
\operatorname{Re} f\left(2^{m} v_{m}\right) & =\sum_{k=0}^{m-1} \operatorname{Re} f\left(2^{k+1} v_{k+1}-2^{k} v_{k}\right) \\
& \leq\left(2^{m}-1\right) \sigma(v)+\delta=2^{m} \operatorname{Re} f(v)+\delta-\sigma(v), \quad m \in \mathbb{N} .
\end{aligned}
$$

Hence, using that $v \in C_{1}$ and $2^{m} v-2^{m} v_{m} \in C_{m+1}$, we have

$$
\begin{aligned}
\inf _{n} \operatorname{Re} g_{0}\left(y_{n}\right) & \leq \inf _{y \in C_{1}} \sigma(y) \leq \sigma(v) \leq \limsup _{m} \operatorname{Re} f\left(2^{m} v-2^{m} v_{m}\right)+\delta \\
& \leq \limsup _{m} \operatorname{Re} f\left(y_{m}\right)+\delta \leq \sigma_{F}+\delta .
\end{aligned}
$$

This contradicts (1).

Remark. The above argument also yields easy proofs of the Simons additive diagonal lemma (cf. [40]) and the Simons inequality (cf. [39. Lemma 2] or 19, Lemma $75])$.

Proof of Lemma 2.1. We first prove that, for each $f \in B_{\mathcal{L}(Y)^{*}}$,

$$
\limsup _{n} \operatorname{Re} f\left(a I_{Y}+b L_{n}+c S\right) \leq 1 \quad \forall b \in B, \quad \forall S \in B_{\mathcal{K}(Y)} .
$$

For any $A \in \mathcal{L}(Y)$, using the weak* compactness of $B_{\mathcal{L}(Y)^{*}}$, we have

$$
\begin{aligned}
\sup \left\{\operatorname{Re} f(A): f \in B_{\mathcal{L}(Y)^{*}}\right\} & =\|A\|=\sup \left\{\operatorname{Re}\left(y \otimes y^{*}\right)(A): y \otimes y^{*} \in S_{Y} \otimes S_{Y^{*}}\right\} \\
& =\max \left\{\operatorname{Re} f(A): f \in \overline{S_{Y} \otimes S_{Y^{*}}} w^{*}\right\} .
\end{aligned}
$$

Hence, by Theorem 2.2, it suffices to prove (3) for each $f \in \overline{S_{Y} \otimes S_{Y^{*}}} w^{*}$. Consider $f=w^{*}-\lim y_{\nu} \otimes y_{\nu}^{*}$, i.e., $y_{\nu}^{*}\left(A y_{\nu}\right) \longrightarrow f(A), A \in \mathcal{L}(Y)$, with $y_{\nu} \in S_{Y}, y_{\nu}^{*} \in S_{Y^{*}}$. By 
passing to a subnet, we may assume that $\left(y_{\nu}^{*}\right)$ converges weak ${ }^{*}$ to some $y^{*} \in B_{Y^{*}}$. From property $M^{*}(a, B, c)$, we get that

$$
\underset{\nu}{\limsup }\left\|a y_{\nu}^{*}+b y^{*}+c S^{*} y^{*}\right\| \leq 1
$$

Hence, for any fixed $n \in \mathbb{N}$,

$$
\begin{aligned}
\mid f\left(a I_{Y}\right. & \left.+b L_{n}+c S\right)\left|=\lim _{\nu}\right| a y_{\nu}^{*}\left(y_{\nu}\right)+b\left(L_{n}^{*} y_{\nu}^{*}\right)\left(y_{\nu}\right)+c\left(S^{*} y_{\nu}^{*}\right)\left(y_{\nu}\right) \mid \\
\leq & \limsup _{\nu}\left|\left(a y_{\nu}^{*}+b y^{*}+c S^{*} y^{*}\right)\left(y_{\nu}\right)\right|+\underset{\nu}{\limsup }\left|\left(b L_{n}^{*}+c S^{*}\right)\left(y_{\nu}^{*}-y^{*}\right)\left(y_{\nu}\right)\right| \\
& +|b| \limsup _{\nu}\left|\left(L_{n}^{*} y^{*}-y^{*}\right)\left(y_{\nu}\right)\right| \leq 1+|b|\left\|L_{n}^{*} y^{*}-y^{*}\right\|,
\end{aligned}
$$

which implies (3) by taking lim $\sup _{n}$.

Let us now fix $S \in B_{\mathcal{K}(Y)}$. We shall show that, for any $n \in \mathbb{N}$ and for any $\epsilon>0$, there exists $K \in \operatorname{conv}\left\{L_{n}, L_{n+1}, \ldots\right\}$ so that

$$
\left\|a I_{Y}+b K+c S\right\| \leq 1+\epsilon \quad \forall b \in B .
$$

Since $\mathcal{K}(Y)$ is separable, the existence of the required $\left(K_{n}\right)_{n=1}^{\infty}$ will then follow by a standard diagonal argument.

To show (4), it is clearly enough to consider the case when $B$ is finite, say $B=$ $\left\{b_{1}, \ldots, b_{m}\right\}$. If $(4)$ is false, then applying the Hahn-Banach separation theorem in the product space $\prod_{k=1}^{m} \mathcal{L}(Y)$ equipped with the maximum norm, we get functionals $f_{1}, \ldots, f_{m} \in \mathcal{L}(Y)^{*}$ with $\left\|f_{1}\right\|+\cdots+\left\|f_{m}\right\|=1$ so that

$$
\begin{aligned}
\sup \left\{\operatorname{Re}\left(f_{1}, \ldots, f_{m}\right)\left(A_{1}, \ldots, A_{m}\right):\right. & \left(A_{1}, \ldots, A_{m}\right) \\
& \left.\in \bar{B}\left(\left(-a I_{Y}-c S, \ldots,-a I_{Y}-c S\right), 1+\epsilon\right)\right\} \\
=-\operatorname{Re} \sum_{k=1}^{m} f_{k}\left(a I_{Y}+c S\right)+1+\epsilon \leq & \operatorname{Re} \sum_{k=1}^{m} f_{k}\left(b_{k} K\right) \quad \forall K \in \operatorname{conv}\left\{L_{n}, L_{n+1}, \ldots\right\}
\end{aligned}
$$

(where $\bar{B}(x, r)$ denotes the closed ball with center $x$ and radius $r$ ). Consequently, by $(3)$,

$$
1+\epsilon \leq \sum_{k=1}^{m} \limsup _{n} \operatorname{Re} f_{k}\left(a I_{Y}+b_{k} L_{n}+c S\right) \leq \sum_{k=1}^{m}\left\|f_{k}\right\|=1,
$$

which is a contradiction.

In Section 3 below, we shall need the following immediate consequence of Lemma 2.1 and Corollary 1.7.

Corollary 2.3. Let $X$ be a Banach space satisfying property $M^{*}(a, B, c)$ with $\max |B|+c>1$. If a separable closed subspace $Y$ of a quotient space of $X$ has an MAI (resp. MCAI) $\left(L_{n}\right)_{n=1}^{\infty}$, then $Y$ also has a shrinking MAI (resp. MCAI) $\left(K_{n}\right)_{n=1}^{\infty}$ with $K_{n} \in \operatorname{conv}\left\{L_{n}, L_{n+1}, \ldots\right\}, n \in \mathbb{N}$, such that

$$
\limsup _{n}\left\|a I_{Y}+b K_{n}+c S\right\| \leq 1 \quad \forall b \in B, \quad \forall S \in B_{\mathcal{K}(Y)} .
$$

Lemma 2.1, together with the remark after it, Lemma 1.1, and Corollary 2.3 yield, for separable Banach spaces, the following characterization of the existence of special shrinking approximations of the identity.

Corollary 2.4. The following assertions are equivalent for a separable Banach space $X$. 
$1^{\circ}$. X has a shrinking $A I$ (resp. $\left.C A I\right)$ and property $M^{*}(a, B, c)$.

$2^{\circ} . X$ has a shrinking MAI (resp. MCAI) $\left(K_{n}\right)_{n=1}^{\infty}$ satisfying

$$
\limsup _{n}\left\|a I_{X}+b K_{n}+c S\right\| \leq 1 \quad \forall b \in B,
$$

for every $S \in B_{\mathcal{K}(X)}$

$3^{\circ}$. For every rank one operator $S \in B_{\mathcal{K}(X)}$, X has a shrinking $A I$ (resp. CAI) $\left(K_{n}\right)_{n=1}^{\infty}$ satisfying (5).

If $\max |B|+c>1$, then $1^{\circ}, 2^{\circ}$, and $3^{\circ}$ are equivalent to the following assertion.

$1^{\circ \circ}$. X has the MAP (resp. MCAP) and property $M^{*}(a, B, c)$.

Remark. If $X^{*}$ is separable and has an AI, then $X$ has a shrinking AI (this easily follows from the principle of local reflexivity) and, as mentioned above, $X$ even has a shrinking MAI. If $X^{*}$ is separable and has a CAI, then $X$ may fail to have a shrinking CAI [18. Moreover, there is a Banach space $X$ failing the MCAP such that all its duals $X^{*}, X^{* *}, \ldots$ have the MCAP and are separable [3].

Casazza and Kalton [4] define a separable Banach space $X$ to have the reverse monotone approximation property $(R M A P)$ provided $X$ has an AI $\left(K_{n}\right)_{n=1}^{\infty}$ with $\lim \left\|I_{X}-K_{n}\right\|=1$. Corollary 2.4 shows, in particular, that $X^{*}$ has the RMAP if $X^{*}$ is separable, has the AP, and satisfies $\lim \sup \left\|x_{n}^{*}-x^{*}\right\| \leq \lim \sup \left\|x_{n}^{*}\right\|$ whenever $\left(x_{n}^{*}\right)_{n=1}^{\infty}$ converges weak* to $x^{*}$ in $X^{*}$.

Note that the equivalence $1^{\circ \circ} \Longleftrightarrow 2^{\circ}$ of Corollary 2.4 is immediate from Lemma 1.1, Corollary 1.7, and Lemma 2.1. The special case of this equivalence for $\left(w M^{*}\right)=$ $M^{*}(1,\{-2\}, 0)$ was established by Lima [31, Theorem 4.2] based on Milman's converse to the Krein-Milman theorem and the Choquet integral representation theorem. The special case of this equivalence for $\left(M^{*}\right)=M^{*}(1,\{-1\}, 1)$ is a well-known consequence of a theorem due to Kalton [27. Theorem 2.4] and the Kalton-Werner theorem stated in the Introduction (the proof of the Kalton-Werner theorem in [28, pp. 144-149] consists in a technical and quite long construction which, departing from a shrinking MCAI $\left(L_{n}\right)_{n=1}^{\infty}$ and using $\left(M^{*}\right)$ to average a certain subsequence of $\left(L_{n}\right)_{n=1}^{\infty}$, gives a shrinking MCAI $\left(K_{n}\right)_{n=1}^{\infty}$ with $\left.\lim \left\|I-2 K_{n}\right\|=1\right)$. Our approach through the Simons sup-lim sup theorem is elementary and much shorter.

\section{Separably determined approximations of the identity. THE MAIN THEOREM}

In the present section, we prove that the existence of the approximations of the identity related to the $M^{*}(a, B, c)$-property is separably determined (see Theorems 3.3 and 3.4 below). This together with results of previous sections will give the main result of the present paper (Theorem 3.5).

We begin by showing that property $M^{*}(a, B, c)$ is separably determined.

Proposition 3.1. If all separable closed subspaces of a Banach space $X$ have property $M^{*}(a, B, c)$, then $X$ has property $M^{*}(a, B, c)$.

Proof. If $X$ fails property $M^{*}(a, B, c)$, then

$$
\limsup _{\nu}\left\|a x_{\nu}^{*}+b x^{*}+c y^{*}\right\|>\limsup _{\nu}\left\|x_{\nu}^{*}\right\|
$$


for some $b \in B, x^{*}, y^{*} \in X^{*}$ with $\left\|y^{*}\right\|<\left\|x^{*}\right\|$, and a bounded net $\left(x_{\nu}^{*}\right)$ converging weak* to $x^{*}$ in $X^{*}$. By passing to a subnet, we may assume that

$$
\inf _{\nu}\left\|a x_{\nu}^{*}+b x^{*}+c y^{*}\right\|>\delta>\sup _{\nu}\left\|x_{\nu}^{*}\right\|
$$

for some $\delta>0$. Choose $x_{0} \in S_{X}$ and $\nu_{1}$ so that $\left\|y^{*}\right\|<\left|x^{*}\left(x_{0}\right)\right|$ and $\left|\left(x_{\nu_{1}}^{*}-x^{*}\right)\left(x_{0}\right)\right|<1$. For $k=1,2, \ldots$, choose inductively $x_{k} \in S_{X}$ and $\nu_{k+1}$ so that

$$
\left|\left(a x_{\nu_{k}}^{*}+b x^{*}+c y^{*}\right)\left(x_{k}\right)\right|>\delta
$$

and

$$
\left|\left(x_{\nu_{k+1}}^{*}-x^{*}\right)\left(x_{0}\right)\right|<1 /(k+1), \ldots,\left|\left(x_{\nu_{k+1}}^{*}-x^{*}\right)\left(x_{k}\right)\right|<1 /(k+1) .
$$

Put $Y=\overline{\operatorname{span}}\left\{x_{0}, x_{1}, \ldots\right\}$. Then $\left\|\left.y^{*}\right|_{Y}\right\|<\left\|\left.x^{*}\right|_{Y}\right\|$ and $x_{\nu_{n}}^{*}(y) \longrightarrow x^{*}(y)$ for all $y \in Y$, but

$$
\limsup _{n}\left\|\left.\left(a x_{\nu_{n}}^{*}+b x^{*}+c y^{*}\right)\right|_{Y}\right\| \geq \delta>\limsup _{n}\left\|\left.x_{\nu_{n}}^{*}\right|_{Y}\right\| .
$$

This means that $Y$ fails property $M^{*}(a, B, c)$.

For the following results, let us recall that, as everywhere, $a, c \geq 0$ and $B \subset \mathbb{K}$ is a compact set.

Lemma 3.2. Let $X$ be a Banach space and $T \in B_{\mathcal{L}(X)}$. If $X$ has the MAP (resp. MCAP) and all its separable closed subspaces $Y$ with an MAI (resp. MCAI) $\left(L_{n}\right)_{n=1}^{\infty}$ have the property that whenever $S \in B_{\mathcal{K}(Y)}$, then there exists a sequence $\left(K_{n}\right)_{n=1}^{\infty}$ in $\operatorname{conv}\left\{L_{1}, L_{2}, \ldots\right\}$ satisfying

$$
\limsup _{n}\left\|\left.a T\right|_{Y}+\left.b T\right|_{Y} K_{n}+c S\right\| \leq 1 \quad \forall b \in B
$$

then, for any $S \in B_{\mathcal{K}(X)}$, there exists an $M A I$ (resp. MCAI) $\left(K_{\alpha}\right)$ of $X$ such that

$$
\limsup _{\alpha}\left\|a T+b T K_{\alpha}+c S\right\| \leq 1 \quad \forall b \in B .
$$

Remark. For the results of this section, only the case $T=I_{X}$ of Lemma 3.2 is needed. The general case will be used in Section 4 below.

Proof of Lemma 3.2. We shall prove the result only in the MCAP case. The MAP case will be evident from the proof below. We shall develop an idea from our paper [36] (where it was used to show that $M$-ideals of compact operators are separably determined).

We denote by $s_{\text {op }}$ the strong operator topology on $\mathcal{L}(X)$. We assume for contradiction that the condition of the lemma is not satisfied: for some $S \in B_{\mathcal{K}(X)}$, there is no such MCAI. Then there are $\epsilon>0$ and a convex $s_{\text {op }}$ neighbourhood $U_{0}$ of $I=I_{X}$ so that

$$
\max _{b \in B}\|a T+b T K+c S\|>1+\epsilon \quad \forall K \in B_{\mathcal{K}(X)} \cap U_{0} .
$$

We clearly may assume that $B$ is finite, say $B=\left\{b_{1}, \ldots, b_{m}\right\}$.

Let $\left(L_{\alpha}\right)_{\alpha \in \mathcal{A}}$ be a net in $B_{\mathcal{K}(X)}$ converging to $I$ in the $s_{\text {op }}$. We shall pick a sequence $\alpha_{1}, \alpha_{2}, \ldots$ in $\mathcal{A}$ and define a separable closed subspace $Y \subset X$ so that $S(X) \subset Y, K_{n}(X) \subset Y$ for all $K_{n}:=L_{\alpha_{n}}, K_{n} y \longrightarrow y$ for all $y \in Y$ and

$$
\max _{1 \leq l \leq m}\left\|\left.\left(a T+b_{l} T K+c S\right)\right|_{Y}\right\|>1+\frac{\epsilon}{2} \quad \forall K \in \operatorname{conv}\left\{K_{1}, K_{2}, \ldots\right\} .
$$

This will contradict the assumption and complete the proof. 
For all $n \in \mathbb{N}$, we denote by $\Lambda_{n}$ a finite $\epsilon /(4 \max |B|+1)$-net in the subset $\left\{\left(\lambda_{1}, \ldots, \lambda_{n}\right): \lambda_{k} \geq 0, \lambda_{1}+\cdots+\lambda_{n}=1\right\}$ of $\ell_{1}^{n}$. We begin by choosing any $K_{1}:=L_{\alpha_{1}} \in U_{0}$ such that $\left\|K_{1} x-x\right\|<1$ for all $x \in S\left(B_{X}\right)$. Let us then assume that a convex $s_{\text {op }}$ neighbourhood $U_{n-1} \subset U_{n-2}$ (where $U_{-1}:=U_{0}$ ) and $K_{n}:=L_{\alpha_{n}} \in U_{n-1}$ have been chosen. We consider $S_{l, \lambda} \in \mathcal{L}(X), l \in\{1, \ldots, m\}$, $\lambda=\left(\lambda_{1}, \ldots, \lambda_{n}\right) \in \Lambda_{n}$, defined by

$$
S_{l, \lambda}=a T+b_{l} T\left(\lambda_{1} K_{1}+\cdots+\lambda_{n} K_{n}\right)+c S,
$$

select $x_{l, \lambda} \in B_{X}$ so that $\left\|S_{l, \lambda} x_{l, \lambda}\right\|>\left\|S_{l, \lambda}\right\|-\epsilon / 4$, and put $C_{n}=\left\{x_{l, \lambda}: \quad l \in\right.$ $\left.\{1, \ldots, m\}, \lambda \in \Lambda_{n}\right\}$. Further, let the product space $\mathcal{L}:=\prod_{l=1}^{m} \mathcal{L}(X)$ be equipped with the maximum norm and also with the product topology $s_{\text {op }} \times \cdots \times s_{\text {op }}$. Denote $F_{n}=(1+\epsilon) B_{\mathcal{L}}-\left\{\left(b_{1} T K, \ldots, b_{m} T K\right): K \in \operatorname{conv}\left\{K_{1}, \ldots, K_{n}\right\}\right\}-\{(c S, \ldots, c S)\}$ and notice that $F_{n}$ is closed in $s_{\text {op }} \times \cdots \times s_{\text {op }}$. Since $(a T, \ldots, a T) \notin F_{n}$ (by (6)), there exists a convex $s_{\text {op }}$ neighbourhood $U_{n} \subset U_{n-1}$ of $I$ so that, for the convex $s_{\text {op }}$ neighbourhood $V_{n}:=U_{n}+\{T-I\}$ of $T$, we have $\left(a V_{n} \times \cdots \times a V_{n}\right) \cap F_{n}=\varnothing$, which implies

$$
\max _{1 \leq l \leq m}\left\|a L+b_{l} T K+c S\right\|>1+\epsilon \quad \forall K \in \operatorname{conv}\left\{K_{1}, \ldots, K_{n}\right\}, \quad \forall L \in V_{n} .
$$

Finally, choose $K_{n+1}:=L_{\alpha_{n+1}} \in U_{n}$ such that

$$
\left\|K_{n+1} x-x\right\|<\frac{1}{n+1} \quad \forall x \in C_{1} \cup \cdots \cup C_{n} \cup S\left(B_{X}\right) \cup K_{1}\left(B_{X}\right) \cup \cdots \cup K_{n}\left(B_{X}\right) .
$$

Let us put $Y=\left\{x \in X: \lim K_{n} x=x\right\}$. It is straightforward to check that $Y$ is a separable closed subspace of $X, S(X) \subset Y$, and $C_{n} \cup K_{n}(X) \subset Y$ for all $n \in \mathbb{N}$. By definition, $K_{n} y \longrightarrow y$ for all $y \in Y$. Moreover, if $K \in \operatorname{conv}\left\{K_{1}, K_{2}, \ldots\right\}$, then $K \in \operatorname{conv}\left\{K_{1}, \ldots, K_{n}\right\}$ for some $n$. Hence, for some $\lambda \in \Lambda_{n}$ and all $l=1, \ldots, m$, we have $\left\|a T+b_{l} T K+c S-S_{l, \lambda}\right\|<\epsilon / 4$. Therefore

$$
\begin{aligned}
\max _{1 \leq l \leq m}\left\|\left.\left(a T+b_{l} T K+c S\right)\right|_{Y}\right\| & >\max _{1 \leq l \leq m}\left\|\left.S_{l, \lambda}\right|_{Y}\right\|-\frac{\epsilon}{4} \\
& >\max _{1 \leq l \leq m}\left\|S_{l, \lambda}\right\|-\frac{\epsilon}{2}>1+\frac{\epsilon}{2}
\end{aligned}
$$

by (7) (recall that $T \in V_{n}$ ). As it was observed above, this completes the proof.

Theorem 3.3. Suppose that $\max |B|+c>1$. If a Banach space $X$ has the $M A P$ (resp. MCAP) and all its separable closed subspaces $Y$ with the MAP (resp. $M C A P)$ have the property that, for any rank one operator $S \in B_{\mathcal{K}(Y)}$, there exists a shrinking $C A I\left(K_{n}\right)_{n=1}^{\infty}$ satisfying

$$
\underset{n}{\limsup }\left\|a I_{Y}+b K_{n}+c S\right\| \leq 1 \quad \forall b \in B,
$$

then, for any $S \in B_{\mathcal{K}(X)}$, there exists a shrinking $M A I$ (resp. MCAI) $\left(K_{\alpha}\right)$ of $X$ such that

$$
\limsup _{\alpha}\left\|a I_{X}+b K_{\alpha}+c S\right\| \leq 1 \quad \forall b \in B .
$$

Proof. First we claim that $X$ has property $M^{*}(a, B, c)$. In fact, let us consider any separable closed subspace $Y$ of $X$. By Proposition 3.1, it suffices to show that $Y$ has property $M^{*}(a, B, c)$. Since $X$ has the $\mathrm{M}(\mathrm{C}) \mathrm{AP}, Y$ is contained in a separable closed subspace $Z$ of $X$ having the M(C)AP (the proof of this fact for the MCAP 
is the same as for the MAP (cf., e.g., [41 p. 606])). Then by Lemma 1.1, $Z$ has $M^{*}(a, B, c)$ and consequently so does $Y$.

Since $X$ has $M^{*}(a, B, c)$ with $\max |B|+c>1$, by Corollary 2.3, it follows from Lemma 3.2 that, for any $S \in B_{\mathcal{K}(X)}$, there exists an $\mathrm{M}(\mathrm{C}) \mathrm{AI}\left(K_{\alpha}\right)$ satisfying $(8 \alpha)$. This $\left(K_{\alpha}\right)$ is shrinking by Corollary 1.7 .

It is known that a Banach space $X$ has the MAP if and only if every separable closed subspace of $X$ is contained in a separable closed subspace $Y$ with the MAP (see [23, Lemma 1 and the proof of Corollary 1] and [24, Lemma 3, (b)], or [41, p. 606]). (The "if" part of this result relies on [32, Lemma 1] and a compactness argument of Lindenstrauss.) We shall use this result to obtain the following theorem.

Theorem 3.4. Suppose that $\max |B|+c>1$. A Banach space $X$ has, for any $S \in B_{\mathcal{K}(X)}$, a shrinking $M A I\left(K_{\alpha}\right)$ satisfying $(8 \alpha)$ if and only if every separable closed subspace of $X$ is contained in a separable closed subspace $Y$ having, for any $S \in B_{\mathcal{K}(Y)}$, a shrinking $M A I\left(K_{n}\right)_{n=1}^{\infty}$ satisfying $(8 n)$.

Proof. If $X$ has, for any $S \in B_{\mathcal{K}(X)}$, a shrinking MAI $\left(K_{\alpha}\right)$ satisfying $(8 \alpha)$, then $X$ has property $M^{*}(a, B, c)$ (cf. Lemma 1.1). Let us consider a separable closed subspace of $X$. It is contained in a separable closed subspace $Y$ with the MAP. By Corollary $2.3, Y$ has a desired MAI $\left(K_{n}\right)_{n=1}^{\infty}$. This proves the necessity part.

For the sufficiency, it is clear - from the result stated before Theorem 3.4 - that $X$ has the MAP. Further, we can apply Theorem 3.3 to get the desired $\left(K_{\alpha}\right)$. In fact, consider any separable closed subspace $Y$ of $X$ with the MAP. Then $Y$ is contained in a separable subspace $Z$ having, for any $S \in B_{\mathcal{K}(Z)}$, the special MAI given by assumption. This implies (cf. Lemma 1.1) that $Z$ has $M^{*}(a, B, c)$. And by Corollary 2.3, $Y$ satisfies the assumption of Theorem 3.3.

Remark. Theorem 3.4 remains valid if one replaces "for any $S$ " by "for any rank one $S^{\prime \prime}$. This is clear from its proof.

Question. We do not know whether Theorem 3.4 remains valid if one replaces MAI by MCAI. We do not know whether it is true that $X$ has the MCAP whenever every separable closed subspace of $X$ is contained in a separable closed subspace having the MCAP. If the last assertion is true, then Theorem 3.4 remains also valid for the MCAI case (as the above proof shows).

We have essentially proved the following (main) result.

Theorem 3.5. Suppose that $\max |B|+c>1$. Then the following assertions are equivalent for a Banach space $X$.

$1^{\circ}$. For any $S \in B_{\mathcal{K}(X)}$, there exists a shrinking MAI (resp. MCAI) $\left(K_{\alpha}\right)$ satisfying $(8 \alpha)$.

$1^{\circ \circ}$. For any rank one operator $S$ with $\|S\| \leq 1$, there exists a shrinking MAI (resp. MCAI) $\left(K_{\alpha}\right)$ satisfying $(8 \alpha)$.

$2^{\circ}$. X has the MAP (resp. MCAP) and property $M^{*}(a, B, c)$.

$3^{\circ} . X$ has the MAP (resp. MCAP) and, for every separable closed subspace $Y$ of $X$ having the MAP (resp. MCAP), there exists a shrinking MAI (resp. $M C A I)\left(K_{n}\right)_{n=1}^{\infty}$ satisfying $(8 n)$ for any $S \in B_{\mathcal{K}(Y)}$.

Proof. The implication $1^{\circ} \Longrightarrow 1^{\circ \circ}$ is obvious. The implications $1^{\circ \circ} \Longrightarrow 2^{\circ}, 2^{\circ} \Longrightarrow$ $3^{\circ}$, and $3^{\circ} \Longrightarrow 1^{\circ}$ are immdiate from Lemma 1.1, Corollary 2.3, and Theorem 3.3, respectively. 
We would like to point out the next immediate corollary.

Corollary 3.6. Suppose that $\max |B|>1$. A Banach space $X$ has a shrinking $M A I$ (resp. MCAI) $\left(K_{\alpha}\right)$ satisfying

$$
\limsup \left\|a I_{X}+b K_{\alpha}\right\| \leq 1 \quad \forall b \in B
$$

if and only if $X$ has the MAP (resp. MCAP) and property $M^{*}(a, B, 0)$; that is,

$$
\limsup _{\nu}\left\|a x_{\nu}^{*}+b x^{*}\right\| \leq \underset{\nu}{\limsup }\left\|x_{\nu}^{*}\right\| \quad \forall b \in B
$$

whenever a bounded net $\left(x_{\nu}^{*}\right)$ converges weak $k^{*}$ to $x^{*}$ in $X^{*}$.

The special case $a=1, B=\{-2\}$ of Corollary 3.6 was proved by Lima 31. Theorem 4.2] for separable $X$ and (with a different argument) for reflexive $X$. This case of Corollary 3.6 gives an internal geometric characterization of the so-called unconditional $\mathrm{M}(\mathrm{C}) \mathrm{AP}$ with adjoint operators (cf. [4], 16]). As it can be seen from Corollary 3.6, the complex version of this unconditional $\mathrm{M}(\mathrm{C}) \mathrm{AP}$ (cf. [16]) is intrinsically characterized by property $M^{*}(1,\{b \in \mathbb{C}:|b+1|=1\}, 0)$. Corollary 3.6 also applies to characterize shrinking MAI related to Lemma 2.2 of [16] through property $M^{*}(a, B, 0)$ with $a>0$ and $B$ compact. Corollary 3.6 will further be developed in Corollary 4.5 below.

Let us finally mention the following immediate conclusion from Corollary 3.6 because it represents the general (i.e., non-separable) version of the Kalton-Werner theorem (cf. the Introduction) giving also its extension to the complex case.

Corollary 3.7. If a Banach space $X$ has the MAP (resp. MCAP) and property $\left(M^{*}\right)$, then $X$ has a shrinking MAI (resp. MCAI) $\left(K_{\alpha}\right)$ satisfying

$$
\lim \left\|I_{X}-(1+\lambda) K_{\alpha}\right\|=1
$$

whenever $|\lambda|=1$.

\section{Applichtions to ideals of COMpaCt AND APPROXimable operators}

According to the terminology in [16], a closed subspace $\mathcal{K} \neq\{0\}$ of a Banach space $\mathcal{L}$ is said to be an ideal in $\mathcal{L}$ if there exists a norm one projection $P$ on $\mathcal{L}^{*}$ with $\operatorname{ker} P=\mathcal{K}^{\perp}=\left\{f \in \mathcal{L}^{*}:\left.f\right|_{\mathcal{K}}=0\right\}$. In this case, we shall say that $P$ is an ideal projection. If $\|P f\|+\|f-P f\|=\|f\|$ for all $f \in \mathcal{L}^{*}$, then $\mathcal{K}$ is called an $M$-ideal. The class of $M$-ideals is extensively studied by many authors (see, e.g., the monograph 20] for results and references). If $\|I-2 P\|=1$, then $\mathcal{K}$ is called a $u$-ideal 4 , and its complex version with $\|I-(1+\lambda) P\|=1$ whenever $|\lambda|=1$ is called an $h$-ideal [16]. A deep study of $u$ - and $h$-ideals was made in [16] (see also [4, 8, 9], 14, [15, 21], 31], 35], 37]). If there are $r, s \in(0,1]$ so that $r\|P f\|+s\|f-P f\| \leq\|f\|$ for all $f \in \mathcal{L}^{*}$, then $\mathcal{K}$ is called an ideal satisfying the $M(r, s)$-inequality. Those ideals of compact operators were recently studied in [2]. Finally, let us recall that every Banach space $X$ is an ideal in $X^{* *}$ with respect to the canonical projection $\pi_{X}$ of $X^{* * *}$ onto $X^{*}$.

As an application of the previous sections, we shall prove the following theorem which, in particular, yields an alternative unified approach to the theories of $M$-, $u$-, and $h$-ideals of compact operators (cf. [20] Chapter VI], together with [28], and [4], [16], [31]). 
Let $a, c \geq 0$ and let $B \subset \mathbb{K}$ be a compact set. We shall need the following natural extension of property $M^{*}(a, B, c)$ from spaces to operators. We say that $T \in B_{\mathcal{L}(X)}$ has property $M^{*}(a, B, c)$ if

$$
\limsup _{\nu}\left\|T^{*}\left(a x_{\nu}^{*}+b x^{*}\right)+c y^{*}\right\| \leq \limsup _{\nu}\left\|x_{\nu}^{*}\right\| \quad \forall b \in B
$$

whenever $x^{*}, y^{*} \in X^{*}$ satisfy $\left\|y^{*}\right\| \leq\left\|x^{*}\right\|$, and $\left(x_{\nu}^{*}\right)$ is a bounded net converging weak* to $x^{*}$ in $X^{*}$.

Theorem 4.1. Let $X$ be a Banach space, let $\mathcal{K}=\mathcal{A}(X)($ resp. $\mathcal{K}=\mathcal{K}(X))$, and let $\mathcal{L}$ be a closed subspace of $\mathcal{L}(X)$ containing $\mathcal{K}$ and $I_{X}$. If $\max |B|+c>1$, then the following assertions are equivalent.

$1^{\circ} . \mathcal{K}$ is an ideal in $\mathcal{L}$ with an ideal projection $P$ such that

$$
\|a f+b P f\|+c\|P f\| \leq\|f\| \quad \forall b \in B, \quad \forall f \in \mathcal{L}^{*},
$$

$X$ is an Asplund space, and $X^{*}=\overline{\operatorname{span}}\left(w^{*}-\operatorname{sexp} B_{X^{*}}\right)$.

$1^{\circ \circ} . \mathcal{K}$ is an ideal in $\mathcal{L}$ with an ideal projection $P$ such that

$$
\|a f+b P f\|+c\|P f\| \leq\|f\| \quad \forall b \in B, \quad \forall f \in \mathcal{L}^{*},
$$

and

$$
\left\|a x^{* * *}+b \pi_{X} x^{* * *}\right\|+c\left\|\pi_{X} x^{* * *}\right\| \leq\left\|x^{* * *}\right\| \quad \forall b \in B, \quad \forall x^{* * *} \in X^{* * *} .
$$

$2^{\circ} . X$ has the $M A P($ resp. $M C A P)$ and every $T \in B_{\mathcal{L}}$ has property $M^{*}(a, B, c)$.

$3^{\circ}$. There exists a shrinking $M A I$ (resp. MCAI) $\left(K_{\alpha}\right)$ such that, for any $S, T \in B_{\mathcal{L}}$

$$
\limsup _{\alpha}\left\|a T+(b T+c S) K_{\alpha}\right\| \leq 1 \quad \forall b \in B .
$$

$4^{\circ}$. There exists a shrinking MAI (resp. MCAI) $\left(K_{\alpha}\right)$ such that, for any $S \in B_{\mathcal{K}(X)}$ and any $T \in B_{\mathcal{L}}$,

$$
\limsup _{\alpha}\left\|a T+b T K_{\alpha}+c S\right\| \leq 1 \quad \forall b \in B .
$$

$5^{\circ}$. For any rank one operator $S$ with $\|S\| \leq 1$ and any $T \in B_{\mathcal{L}}$, there exists a shrinking MAI (resp. MCAI) $\left(K_{\alpha}\right)$ satisfying (9).

Lemma 4.2. Let $X$ be a Banach space and $T \in B_{\mathcal{L}(X)}$.

(a) If, for any rank one operator $S$ with $\|S\| \leq 1$, there exists a net $\left(K_{\alpha}\right) \subset \mathcal{K}(X)$ such that $K_{\alpha}^{*} x^{*} \longrightarrow T^{*} x^{*}, x^{*} \in X^{*}$, and

$$
\limsup _{\alpha}\left\|a T+b K_{\alpha}+c S\right\| \leq 1 \quad \forall b \in B,
$$

then $T$ has property $M^{*}(a, B, c)$.

(b) Suppose that $X$ has the MAP (resp. MCAP). If $X$ and $T$ have property $M^{*}(a, B, c)$ with $\max |B|+c>1$, then, for any $S \in B_{\mathcal{K}(X)}$, there exists a shrinking MAI (resp. MCAI) ( $\left.K_{\alpha}\right)$ satisfying (9).

Proof. Replacing $I_{X}$ by $T$ in the proof of Lemma 1.1 gives the proof of $(a)$.

Replacing $I_{Y}$ by $\left.T\right|_{Y}$ in the proof of Lemma 2.1 essentially gives the following fact. Let $Y$ be a closed subspace of a Banach space $X$ and let $T \in B_{\mathcal{L}(X)}$ have property $M^{*}(a, B, c)$. If there exists a sequence $L_{n}: Y \longrightarrow X, n \in \mathbb{N}$, of compact 
operators such that $L_{n}^{*} x^{*} \longrightarrow\left(\left.T\right|_{Y}\right)^{*} x^{*}, x^{*} \in X^{*}$, then, for any compact operator $S: Y \longrightarrow X,\|S\| \leq 1$, there exist $K_{n} \in \operatorname{conv}\left\{L_{n}, L_{n+1}, \ldots\right\}, n \in \mathbb{N}$, so that

$$
\limsup _{n}\left\|\left.a T\right|_{Y}+b K_{n}+c S\right\| \leq 1 \quad \forall b \in B .
$$

We shall apply Lemma 3.2. Consider any separable closed subspace $Y$ of $X$ with an $\mathrm{M}(\mathrm{C}) \mathrm{AI}\left(L_{n}\right)_{n=1}^{\infty}$ and an operator $S \in B_{\mathcal{K}(Y)}$. Since $X$ satisfies property $M^{*}(a, B, c)$ with $\max |B|+c>1$, by Corollary 1.7, $\left(L_{n}\right)_{n=1}^{\infty}$ is shrinking and therefore $\left(\left.T\right|_{Y} L_{n}\right)^{*} x^{*} \longrightarrow\left(\left.T\right|_{Y}\right)^{*} x^{*}, x^{*} \in X^{*}$. By the above fact, there are $K_{n} \in$ $\operatorname{conv}\left\{L_{n}, L_{n+1}, \ldots\right\}, n \in \mathbb{N}$, so that

$$
\limsup _{n}\left\|\left.a T\right|_{Y}+\left.b T\right|_{Y} K_{n}+c S\right\| \leq 1 \quad \forall b \in B .
$$

An immediate application of Lemma 3.2 together with Corollary 1.7 completes the proof of $(b)$.

Proof of Theorem 4.1. We shall need the following well-known result of J. Johnson 22]. Let $\left(K_{\alpha}\right)$ be a shrinking $\mathrm{M}(\mathrm{C}) \mathrm{AI}$ for $X$. Then, by passing to a subnet of $\left(K_{\alpha}\right)$, one may assume that $\lim _{\alpha} f\left(T K_{\alpha}\right)$ exists for all $f \in \mathcal{L}^{*}$ and $T \in \mathcal{L}$, and $P: \mathcal{L}^{*} \longrightarrow \mathcal{L}^{*}$ defined by

$$
(P f)(T)=\lim _{\alpha} f\left(T K_{\alpha}\right), \quad f \in \mathcal{L}^{*}, \quad T \in \mathcal{L},
$$

is a norm one projection with $\operatorname{ker} P=\mathcal{K}^{\perp}$ (this means that $\mathcal{K}$ is an ideal in $\mathcal{L}$ and $P$ is an ideal projection).

On the other hand, if $\mathcal{K}$ is an ideal in $\mathcal{L}, X$ is an Asplund space, and $X^{*}=$ $\overline{\operatorname{span}}\left(w^{*}-\operatorname{sexp} B_{X^{*}}\right)$, then, by [2, Proposition 3.2], $X$ admits a shrinking M(C)AI $\left(K_{\alpha}\right)$ and the ideal projection is unique.

$1^{\circ} \Longrightarrow 3^{\circ}$. By the above, $X$ admits a shrinking $\mathrm{M}(\mathrm{C}) \mathrm{AI}\left(K_{\alpha}\right)$ which may be assumed to satisfy (10).

The main idea of the argument below is due to $\mathrm{W}$. Werner [43, proof of Theorem 3.5] who applied it to studying inner $M$-ideals in unital Banach algebras. However, we shall not employ Banach algebra techniques but follow [2, proof of Theorem 3.1].

For $T \in \mathcal{L}$, denote by $L_{T}$ the operator on $\mathcal{L}$ defined by $L_{T}(S)=T S, S \in \mathcal{L}$. We consider the set of all $\beta=(\Phi, F, H, \epsilon)$, where $\Phi \subset \mathcal{L}^{* *}, F \subset \mathcal{L}^{*}, H \subset \mathcal{L}$ are finite dimensional subspaces, $\Phi$ containing $P^{*} I$, and $\epsilon>0$, directed in a natural way. As in [2, proof of Theorem 3.1], using a version of the principle of local reflexivity, due to Behrends [1], we have, for every $\beta$, an operator

$$
T_{\beta}: \operatorname{span}\left(\Phi \cup\left\{L_{T}^{* *} \phi: T \in H, \phi \in \Phi\right\}\right) \longrightarrow \mathcal{L}
$$

so that

$$
\begin{aligned}
& T_{\beta} T=T \quad \forall T \in \Phi \cup \mathcal{L}, \\
&\left\|T_{\beta} \phi\right\| \leq(1+\epsilon)\|\phi\| \forall \phi \in \Phi, \\
&\left\|T T_{\beta}\left(P^{*} I\right)-T_{\beta}\left(P^{*} T\right)\right\| \leq \epsilon\|T\| \forall T \in H,
\end{aligned}
$$

(note that $P^{*} T=L_{T}^{* *}\left(P^{*} I\right)$ ), and

$$
\lim _{\beta} f\left(T_{\beta}\left(P^{*} I\right)\right)=\left(P^{*} I\right)(f) \quad \forall f \in \mathcal{L}^{*} .
$$

Also since

$$
\lim _{\alpha} f\left(K_{\alpha}\right)=\left(P^{*} I\right)(f) \quad \forall f \in L^{*}
$$


(cf. (10)), after switching to the product index set $\{(\alpha, \beta)\}$ (with the product ordering) and passing to convex combinations, we may assume that

$$
\left\|K_{\alpha}-T_{\alpha}\left(P^{*} I\right)\right\| \longrightarrow 0 .
$$

Consider $S, T \in B_{\mathcal{L}}$. The above conditions easily yield that, for any $b \in B$,

$$
\begin{aligned}
\underset{\alpha}{\limsup \left\|a T+(b T+c S) K_{\alpha}\right\|} & =\limsup _{\alpha}\left\|a T_{\alpha} T+(b T+c S) T_{\alpha}\left(P^{*} I\right)\right\| \\
& =\limsup _{\alpha}\left\|T_{\alpha}\left(a T+P^{*}(b T+c S)\right)\right\| \\
& \leq\left\|a T+b P^{*} T+c P^{*} S\right\| .
\end{aligned}
$$

The last norm is not greater than 1 . In fact, the inequality of $1^{\circ}$ means that the operator

$$
f \longmapsto(a f+b P f, c P f)
$$

from $\mathcal{L}^{*}$ to $\mathcal{L}^{*} \oplus_{1} \mathcal{L}^{*}$ has norm $\leq 1$. Since its adjoint also has norm $\leq 1$, we have, in particular, that

$$
\left\|a T+b P^{*} T+c P^{*} S\right\| \leq \max \{\|T\|,\|S\|\} \leq 1 .
$$

$3^{\circ} \Longrightarrow 4^{\circ}$ is clear because $\left\|S-S K_{\alpha}\right\|=\left\|\left(K_{\alpha}^{*}-I_{X^{*}}\right) S^{*}\right\| \longrightarrow 0$ whenever $S \in \mathcal{K}(X)$ and the existence of an MAI for $X$ implies that $\mathcal{A}(X)=\mathcal{K}(X)$ (the last fact is well known and easy to prove (cf., e.g., [33], p. 32).

$4^{\circ} \Longrightarrow 5^{\circ}$ is obvious.

$5^{\circ} \Longrightarrow 2^{\circ}$ immediately follows from Lemma $4.2,(a)$.

$2^{\circ} \Longrightarrow 1^{\circ \circ}$. We first note that $\pi_{X}$ satisfies the required inequality because $X$ (or equivalently $I_{X}$ ) has property $M^{*}(a, B, c)$ (see Proposition 1.3). Further, let $\left(L_{\alpha}\right)$ denote an $\mathrm{M}(\mathrm{C}) \mathrm{AI}$ for $X$. According to a well-known result of J. Johnson [22] (this is the symmetric version of the result used in the beginning of the proof of Theorem 4.1), by passing to a subnet of $\left(L_{\alpha}\right)$, one may assume that $\lim _{\alpha} f\left(L_{\alpha} T\right)$ exists for all $f \in \mathcal{L}^{*}$ and $T \in \mathcal{L}$, and $\mathcal{K}$ is an ideal in $\mathcal{L}$ with respect to the ideal projection $P$ defined by

$$
(P f)(T)=\lim _{\alpha} f\left(L_{\alpha} T\right), \quad f \in \mathcal{L}^{*}, \quad T \in \mathcal{L} .
$$

Let us fix $b \in B$ and $f \in \mathcal{L}^{*}$. For given $\epsilon>0$, we choose $S \in B_{\mathcal{K}}$ (using (11) here) and $T \in B_{\mathcal{L}}$ so that

$$
\|a f+b P f\|+c\|P f\|-\epsilon \leq(a f+b P f)(T)+c f(S) .
$$

By Lemma 4.2, (b), for $S$ and $T$, there exists a shrinking $\mathrm{M}(\mathrm{C}) \mathrm{AI}\left(K_{\alpha}\right)$ satisfying (9). By the above, we also may assume that $\left(K_{\alpha}\right)$ satisfies (10). Consequently,

$$
\|a f+b P f\|+c\|P f\|-\epsilon \leq \lim _{\alpha} f\left(a T+b T K_{\alpha}+c S\right) \leq\|f\| .
$$

Since $\epsilon$ is arbitrary, this finishes the proof.

$1^{\circ 0} \Longrightarrow 1^{\circ}$. It suffices to show that $r(V) \leq 1 /(\max |B|+c)$ for any proper closed subspace $V$ of $X^{*}$ because then $X$ is an Asplund space and $X^{*}=\overline{\operatorname{span}}\left(w^{*}\right.$-sexp $\left.B_{X^{*}}\right)$ by the proof of Corollary 1.6. As in the proof of Proposition 1.5, we may assume that $V=\operatorname{ker} x^{* *}$ where $x^{* *} \in S_{X^{* *}}$. Let $\beta=\max |B|=b \operatorname{sgn} b$ for some $b \in B$. We clearly have the inequality

$$
\left\|\left(a \operatorname{sgn} b+\beta \pi_{X}+c \pi_{X}\right) x^{* * *}\right\| \leq\left\|x^{* * *}\right\| \quad \forall x^{* * *} \in X^{* * *},
$$


and therefore

$$
\left\|I_{X^{* * *}}+\frac{\beta+c}{a \operatorname{sgn} b} \pi_{X}\right\| \leq \frac{1}{a} .
$$

By [16] Proposition 2.3], this condition implies the existence of a net $\left(x_{\nu}\right)$ in $B_{X}$ converging weak* to $x^{* *}$ in $X^{* *}$ such that

$$
\underset{\nu}{\limsup }\left\|x^{* *}+\frac{\beta+c}{a \operatorname{sgn} b} x_{\nu}\right\| \leq \frac{1}{a} .
$$

We now continue similarly to [16, the proof of Proposition 2.7]:

$$
\begin{aligned}
(\beta+c) r(V) & \leq(\beta+c) \inf _{\nu} \sup _{x^{*} \in B_{V}}\left|x^{*}\left(x_{\nu}\right)\right| \\
& =a \inf _{\nu} \sup _{x^{*} \in B_{V}}\left|x^{* *}\left(x^{*}\right)+x^{*}\left(\frac{\beta+c}{a \operatorname{sgn} b} x_{\nu}\right)\right| \leq 1
\end{aligned}
$$

by the above inequality. This completes the proof.

Remark 1. (It concerns assertions $1^{\circ}$ and $1^{\circ \circ}$ of Theorem 4.1.) If $\mathcal{K}$ is an $M$-ideal in $\mathcal{L}$, then $X$ is an $M$-ideal in $X^{* *}$ (i.e., $\left\|x^{* * *}-\pi_{X} x^{* * *}\right\|+\left\|\pi_{X} x^{* * *}\right\|=\left\|x^{* * *}\right\|, x^{* * *} \in$ $X^{* * *}$ ). This can be shown by quite a direct application of the 3-ball property (cf. 30] or 20, p. 291]). Further, if $X$ is an $M$-ideal in $X^{* *}$, then $X$ is an Asplund space and $X^{*}=\overline{\operatorname{span}}\left(w^{*}\right.$-sexp$\left.B_{X^{*}}\right)$ (cf., e.g., 20, pp. 126 and 127]). More generally (cf. 2, Lemma 2.3, Proposition 2.1], relying on a different argument, one can show that $X$ is an Asplund space and $X^{*}=\overline{\operatorname{span}}\left(w^{*}\right.$-sexp $\left.B_{X^{*}}\right)$ whenever $\mathcal{K}$ is an ideal satisfying the $M(r, s)$-inequality in $\mathcal{L}$ for some $r, s \in(0,1]$ with $r+s / 2>1$. On the other hand, for the sake of contrast, let us point out that, e.g., $\mathcal{K}\left(\ell_{1}\right)$ is a $u$ ideal in $\mathcal{L}\left(\ell_{1}\right)$. [In fact, the ideal projection defined by the procedure of J. Johnson (described in the proof of $2^{\circ} \Longrightarrow 1^{\circ \circ}$ ), departing from the sequence of the natural projections associated to the unit vector basis of $\ell_{1}$, provides the $u$-ideal property for $\mathcal{K}\left(\ell_{1}\right)$ in $\mathcal{L}\left(\ell_{1}\right)$.]

Remark 2. (It concerns assertion $2^{\circ}$ of Theorem 4.1.) If $X$ has property $\left(M^{*}\right)=$ $M^{*}(1,\{-1\}, 1)$, then (as it is essentially shown in [27, Lemma 2.2]), every $T \in$ $B_{\mathcal{L}(X)}$ has property $\left(M^{*}\right)$. Obviously, every $T \in B_{\mathcal{L}(X)}$ has property $M^{*}(a, B, 0)$ whenever $X$ has $M^{*}(a, B, 0)$. It can also be shown that every $T \in B_{\operatorname{span}\left(\mathcal{K}(X) \cup\left\{I_{X}\right\}\right)}$ has property $M^{*}(a,\{-a\}, c)$ whenever $X$ has $M^{*}(a,\{-a\}, c)$. [For a proof, let us consider $T=K+\lambda I$ with $K \in \mathcal{K}(X)$ and $\|T\| \leq 1$. Note that $|\lambda| \leq 1$ because, otherwise, $K$ would be invertible. Hence $\lambda=r e^{i \phi}$ with $r \in[0,1]$. Let $x^{*}, y^{*} \in X^{*}$ satisfy $\left\|y^{*}\right\| \leq\left\|x^{*}\right\|$ and let $\left(x_{\nu}^{*}\right)$ be a bounded net converging weak ${ }^{*}$ to $x^{*}$ in $X^{*}$. Then, using the property $M^{*}(a,\{-a\}, c)$ for $X$ (which clearly implies that $c \leq 1$ ), we get

$$
\begin{aligned}
& \lim \sup \left\|T^{*}\left(a x_{\nu}^{*}-a x^{*}\right)+c y^{*}\right\|=\lim \sup \left\|r e^{i \phi}\left(a x_{\nu}^{*}-a x^{*}\right)+c y^{*}\right\| \\
& \leq r \limsup _{\nu}\left\|a x_{\nu}^{*}-a x^{*}+c e^{-i \phi} y^{\nu}\right\|+(1-r) c\left\|y^{*}\right\| \\
& \left.\leq r \lim \sup \left\|x_{\nu}^{*}\right\|+(1-r)\left\|x^{*}\right\| \leq \lim \sup \left\|x_{\nu}^{*}\right\| .\right]
\end{aligned}
$$

We do not know whether the last claim can be extended to all $T \in B_{\mathcal{L}(X)}$.

In view of the above remarks, the next three Corollaries 4.3-4.5 are immediate from Theorems 4.1 and 3.5. [Concerning condition $4^{\circ}$ of Corollary 4.5 , let us recall (cf. the proof of Theorem 3.3) that if $X$ has the MCAP, then any separable closed 
subspace of $X$ is contained in a separable closed subspace $Y$ of $X$ having the MCAP. Hence, if $Y^{*}$ is separable for all such subspaces $Y$, then $X$ is an Asplund space.]

Corollary 4.3. The following assertions are equivalent for a Banach space $X$.

$1^{\circ} . \mathcal{K}(X)$ is an $M$-ideal in $\mathcal{L}(X)$.

$2^{\circ} . \mathcal{K}(X)$ is an $M$-ideal in $\operatorname{span}\left(\mathcal{K}(X) \cup\left\{I_{X}\right\}\right)$.

$3^{\circ} . X$ has the $M C A P$ and property $\left(M^{*}\right)$.

$4^{\circ}$. There exists a shrinking MCAI $\left(K_{\alpha}\right)$ such that

$$
\limsup _{\alpha}\left\|T\left(I_{X}-K_{\alpha}\right)+S K_{\alpha}\right\| \leq 1 \quad \forall S, T \in B_{\mathcal{L}(X)} .
$$

$5^{\circ}$. There exists a shrinking $M C A I\left(K_{\alpha}\right)$ such that

$$
\limsup _{\alpha}\left\|T\left(I_{X}-K_{\alpha}\right)+S\right\| \leq 1 \quad \forall S \in B_{\mathcal{K}(X)}, \quad \forall T \in B_{\mathcal{L}(X)} .
$$

$6^{\circ}$. There exists a shrinking $M C A I\left(K_{\alpha}\right)$ such that

$$
\limsup _{\alpha}\left\|I_{X}-K_{\alpha}+S\right\| \leq 1 \quad \forall S \in B_{\mathcal{K}(X)} .
$$

$7^{\circ}$. For any rank one operator $S$ with $\|S\| \leq 1$, there exists a shrinking $M C A I$ $\left(K_{\alpha}\right)$ satisfying

$$
\limsup _{\alpha}\left\|I_{X}-K_{\alpha}+S\right\| \leq 1
$$

$8^{\circ} . X$ has the $M C A P$ and $\mathcal{K}(Y)$ is an $M$-ideal in $\mathcal{L}(Y)$ for all separable closed subspaces $Y$ of $X$ having the $M C A P$.

The equivalences $1^{\circ} \Longleftrightarrow 4^{\circ}$ and $1^{\circ} \Longleftrightarrow 5^{\circ}$ of Corollary 4.3 are essentially due to $\mathrm{W}$. Werner 43 and $\mathrm{D}$. Werner [42, respectively. For separable $X$, the equivalences $1^{\circ} \Longleftrightarrow 2^{\circ} \Longleftrightarrow 6^{\circ}$ and $1^{\circ} \Longleftrightarrow 3^{\circ}$ are, respectively, due to Kalton [27] and Kalton and D. Werner ([27], [28]). For reflexive $X$, the equivalence $1^{\circ} \Longleftrightarrow 3^{\circ}$ was established by Lima [31]. The extensions of $1^{\circ} \Longleftrightarrow 2^{\circ} \Longleftrightarrow 6^{\circ}$ and $1^{\circ} \Longleftrightarrow 3^{\circ}$ to arbitrary (nonseparable) $X$ were proved by the author, respectively, in [34] (cf. also [20, p. 299]) and [36] using methods other than those in this paper. The equivalence $1^{\circ} \Longleftrightarrow 7^{\circ}$ is new and $1^{\circ} \Longleftrightarrow 8^{\circ}$ is due to the author [36], where it was proved relying on the theory of $M$-ideals of compact operators [20 Chapter VI].

Corollary 4.4. Let $X$ be a Banach space and let $\mathcal{I}(X)=\operatorname{span}\left(\mathcal{K}(X) \cup\left\{I_{X}\right\}\right)$. If $r, s \in(0,1]$ satisfy $r+s / 2>1$, then the following assertions are equivalent.

$1^{\circ} . \mathcal{K}(X)$ is an ideal satisfying the $M(r, s)$-inequality in $\mathcal{I}(X)$.

$2^{\circ}$. X has the $M C A P$ and property $M^{*}(s,\{-s\}, r)$.

$3^{\circ}$. There exists a shrinking $M C A I\left(K_{\alpha}\right)$ such that

$$
\limsup _{\alpha}\left\|s T\left(I_{X}-K_{\alpha}\right)+r S K_{\alpha}\right\| \leq 1 \quad \forall S, T \in B_{\mathcal{I}(X)} .
$$

$4^{\circ}$. For any rank one operator $S$ with $\|S\| \leq 1$, there exists a shrinking MCAI $\left(K_{\alpha}\right)$ satisfying

$$
\limsup _{\alpha}\left\|s\left(I_{X}-K_{\alpha}\right)+r S\right\| \leq 1 .
$$

$5^{\circ} . X$ has the $M C A P$ and $\mathcal{K}(Y)$ is an ideal satisfying the $M(r, s)$-inequality in $\mathcal{I}(Y)$ for all separable closed subspaces $Y$ of $X$ having the $M C A P$.

The equivalence $1^{\circ} \Longleftrightarrow 3^{\circ}$ of Corollary 4.4 is due to [2]. 
Corollary 4.5. If $\max |B|>1$, then the following assertions are equivalent for a Banach space $X$.

$1^{\circ} . \mathcal{K}(X)$ is an ideal in $\mathcal{L}(X)$ with an ideal projection $P$ such that $\| a I_{\mathcal{L}(X)^{*}}+$ $b P \| \leq 1$ for all $b \in B, X$ is an Asplund space, and $X^{*}=\overline{\operatorname{span}}\left(w^{*}-\operatorname{sexp} B_{X^{*}}\right)$.

$1^{\circ 0} \cdot \mathcal{K}(X)$ is an ideal in $\mathcal{L}(X)$ with an ideal projection $P$ such that $\| a I_{\mathcal{L}(X)^{*}}+$ $b P \| \leq 1$ for all $b \in B$, and $\left\|a I_{X^{* * *}}+b \pi_{X}\right\| \leq 1$ for all $b \in B$.

$2^{\circ}$. X has the $M C A P$ and property $M^{*}(a, B, 0)$; that is,

$$
\underset{\nu}{\limsup }\left\|a x_{\nu}^{*}+b x^{*}\right\| \leq \underset{\nu}{\limsup }\left\|x_{\nu}^{*}\right\| \quad \forall b \in B
$$

whenever a bounded net $\left(x_{\nu}^{*}\right)$ converges weak $k^{*}$ to $x^{*}$ in $X^{*}$.

$3^{\circ}$. There exists a shrinking $M C A I\left(K_{\alpha}\right)$ satisfying

$$
\limsup _{\alpha}\left\|a I_{X}+b K_{\alpha}\right\| \leq 1 \quad \forall b \in B .
$$

$4^{\circ} . X$ is an Asplund space having the MCAP and every separable closed subspace $Y$ of $X$ having the $M C A P$ satisfies the following: $\mathcal{K}(Y)$ is an ideal in $\mathcal{L}(Y)$ with an ideal projection $P$ such that $\left\|a I_{\mathcal{L}(Y)^{*}}+b P\right\| \leq 1$ for all $b \in B$ and $Y^{*}=\overline{\operatorname{span}}\left(w^{*}-\operatorname{sexp} B_{Y^{*}}\right)$.

According to a theorem of Kalton [27, if $\mathcal{K}(X)$ is an $M$-ideal in $\mathcal{L}(X)$ and $Y$ is a closed subspace of a quotient space of $X$, then $\mathcal{K}(Y)$ is an $M$-ideal in $\mathcal{L}(Y)$ if and only if $Y$ has the MCAP (see [27] for the case of separable $X$ and 34] or [20, p. $301]$ for the general case). Since property $M^{*}(a, B, c)$ is inherited by subspaces of quotient spaces (cf. Section 1), this theorem and analogous results (with obvious modifications) for ideals satisfying the $M(r, s)$-inequality and for $u$-and $h$-ideals are immediate from Corollaries 4.3, 4.4 and 4.5 (cf. resp. equivalences $1^{\circ} \Longleftrightarrow 3^{\circ}$, $1^{\circ} \Longleftrightarrow 2^{\circ}$, and $1^{\circ} \Longleftrightarrow 2^{\circ}$ of these corollaries).

The equivalence $2^{\circ} \Longleftrightarrow 3^{\circ}$ of Corollary 4.5 is contained in Corollary 3.6 above (see also bibliographical references after Corollary 3.6). The implication $1^{\circ} \Longrightarrow 3^{\circ}$ can also be deduced from [16, Lemma 2.2] where ideal projections $P$ satisfying $\|a I+b P\| \leq 1, b \in B$, were characterized in the general setting.

Let us remark that if $X$ is reflexive, then $X$ is an Asplund space and $X^{*}=$ $\overline{\operatorname{span}}\left(w^{*}-\operatorname{sexp} B_{X^{*}}\right)$. The most important particular cases of Corollary 4.5 are those concerning $u$-ideals and $h$-ideals of compact operators; that is, the cases when $a=1$, $B=\{-2\}$ and $a=1, B=\{-(1+\lambda): \lambda \in \mathbb{C},|\lambda|=1\}$. For separable reflexive $X$ having the approximation property and $u$-ideals, the equivalence $1^{\circ} \Longleftrightarrow 3^{\circ}$ of Corollary 4.5 is essentially due to Casazza and Kalton [4, Theorem 3.9]. It was extended to reflexive $X$ by Lima [31, Corollary 4.4]. For separable reflexive $X$ and both $u$ - and $h$-ideals, the equivalence $1^{\circ} \Longleftrightarrow 3^{\circ}$ was proved in [16, Theorem 8.3, $(a)]$ by Godefroy, Kalton, and Saphar using the fact that the points of Fréchet smoothness form a dense $G_{\delta}$ set in both $X$ and $X^{*}$. By similar arguments, also applying the theory of hermitian operators, the equivalence $1^{\circ \circ} \Longleftrightarrow 3^{\circ}$ was essentially proved in [16, Theorem 8.3, (b)] for $h$-ideals under the separability assumption of $X^{*}$. We would like to point out Corollary 4.6 below as an improvement of these results.

Let us recall [16] that $X$ is said to be a strict $u$-ideal in $X^{* *}$ whenever $X$ is a $u$-ideal in $X^{* *}$ with respect to an ideal projection $P$ such that $\operatorname{ran} P$ is a norming subspace of $\left(X^{* *}\right)^{*}$. By [16, Proposition 5.2, Theorem 6.6], an Asplund space $X$ is a strict $u$-ideal (resp. an $h$-ideal) in $X^{* *}$ if and only if $\left\|I_{X^{* * *}}-2 \pi_{X}\right\|=1$ (resp. $\left\|I_{X^{* * *}}-(1+\lambda) \pi_{X}\right\|=1$ for $\left.|\lambda|=1\right)$. From this, Corollary 4.5 , and the fact that 
any MCAI for $X$ is shrinking whenever $X^{*}=\overline{\operatorname{span}}\left(w^{*}-\operatorname{sexp} B_{X^{*}}\right)$ (cf. the proof of Corollary 1.7), the following is immediate.

Corollary 4.6. The following assertions are equivalent for an Asplund space $X$.

$1^{\circ}$. $\mathcal{K}(X)$ is a u-ideal (resp. an $h$-ideal) in $\mathcal{L}(X)$ and $X$ is a strict $u$-ideal (resp. an $h$-ideal) in $X^{* *}$.

$2^{\circ}$. There exists a shrinking $M C A I\left(K_{\alpha}\right)$ satisfying $\lim _{\alpha}\left\|I_{X}-2 K_{\alpha}\right\|=1$ (resp. $\lim _{\alpha}\left\|I_{X}-(1+\lambda) K_{\alpha}\right\|=1$ for $\left.|\lambda|=1\right)$.

If, in addition, $X^{*}=\overline{\operatorname{span}}\left(w^{*}-\operatorname{sexp} B_{X^{*}}\right)$, then $1^{\circ}$ and $2^{\circ}$ are equivalent to the following assertions.

$1^{\circ \circ}$. $\mathcal{K}(X)$ is a u-ideal (resp. an h-ideal) in $\mathcal{L}(X)$.

$2^{\circ \circ}$. There exists an $M C A I\left(K_{\alpha}\right)$ satisfying $\lim _{\alpha}\left\|I_{X}-2 K_{\alpha}\right\|$ (resp. $\lim _{\alpha} \| I_{X}-$ $(1+\lambda) K_{\alpha} \|=1$ for $\left.|\lambda|=1\right)$.

Corollaries 4.3-4.6, with the obvious modifications (see Theorem 4.1 and recall that $\mathcal{A}(X)=\mathcal{K}(X)$ whenever $X$ has an MAI), remain valid for the space $\mathcal{A}(X)$ instead of $\mathcal{K}(X)$. The $\mathcal{A}(X)$ versions of these corollaries together with Theorem 3.4 yield the next results (from which in Corollary 4.9, one also needs the observation that the equality $\left\|I_{X^{* * *}}-(1+\lambda) \pi_{X}\right\|=1$ with $|\lambda|=1$ for $X$ is shared by subspaces of $X$ ) about separably determined ideals of approximable operators.

Corollary 4.7. Let $X$ be a Banach space. Then $\mathcal{A}(X)$ is an $M$-ideal in $\mathcal{L}(X)$ if and only if every separable closed subspace of $X$ is contained in a separable closed subspace $Y$ for which $\mathcal{A}(Y)$ is an $M$-ideal in $\mathcal{L}(Y)$.

Corollary 4.8. Let $X$ be a Banach space and let $\mathcal{I}(X)=\operatorname{span}\left(\mathcal{A}(X) \cup\left\{I_{X}\right\}\right)$. If $r, s \in(0,1]$ satisfy $r+s / 2>1$, then $\mathcal{A}(X)$ is an ideal satisfying the $M(r, s)$ inequality in $\mathcal{I}(X)$ if and only if every separable closed subspace of $X$ is contained in a separable closed subspace $Y$ for which $\mathcal{A}(Y)$ is an ideal satisfying the $M(r, s)$ inequality in $\mathcal{I}(Y)$.

Corollary 4.9. Let $X$ be an Asplund space which is a strict u-ideal (resp. an $h$ ideal) in $X^{* *}$. Then $\mathcal{A}(X)$ is a u-ideal (resp. an h-ideal) in $\mathcal{L}(X)$ if and only if every separable closed subspace of $X$ is contained in a separable closed subspace $Y$ for which $\mathcal{A}(Y)$ is a u-ideal (resp. an h-ideal) in $\mathcal{L}(Y)$.

Question. We do not know whether Corollaries 4.7-4.9 hold for compact operators (cf. the Question after Theorem 3.4).

\section{REFERENCES}

1. E. Behrends, A generalization of the principle of local reflexivity, Rev. Roumaine Math. Pures Appl. 31 (1986), 293-296. MR 88a:46011

2. J. C. Cabello, E. Nieto, and E. Oja, On ideals of compact operators satisfying the $M(r, s)$-inequality, J. Math. Anal. Appl. 220 (1998), 334-348. MR 99f:46018

3. P. G. Casazza and H. Jarchow, Self-induced compactness in Banach spaces, Proc. Roy. Soc. Edinburgh Sect. A 126 (1996), 355-362. MR 97a:46010

4. P. G. Casazza and N. J. Kalton, Notes on approximation properties in separable Banach spaces, Geometry of Banach Spaces. Proc. Conf. Strobl (1989) (P. F. X. Müller and W. Schachermayer, eds.), London Math. Soc. Lecture Note Series, vol. 158, Cambridge Univ. Press, 1990, pp. 49-63. MR 92d:46022

5. Ch.-M. Cho and W. B. Johnson, A characterization of subspaces $X$ of $\ell_{p}$ for which $K(X)$ is an $M$-ideal in $L(X)$, Proc. Amer. Math. Soc. 93 (1985), 466-470. MR 86h:46026

6. J. Diestel and J. J. Uhl, Vector Measures, Mathematical Surveys 15, Amer. Math. Soc., Providence, Rhode Island, 1977. MR 56:12216 
7. D. van Dulst and I. Singer, On Kadec-Klee norms on Banach spaces, Studia Math. 54 (1975), 205-211. MR 52:14937

8. G. Emmanuele and K. John, Some remarks on the position of the space $K(X, Y)$ inside the space $W(X, Y)$, New Zealand J. Math. 26 (1997), 183-189. |MR 98m:46024

9. G. Emmanuele and T. S. S. R. K. Rao, Spaces of Bochner integrable functions and spaces of representable operators as $\mathfrak{U}$-ideals, Quart. J. Math. Oxford (2) 48 (1997), 467-478. MR 99a:46066

10. T. Figiel and W. B. Johnson, The approximation property does not imply the bounded approximation property, Proc. Amer. Math. Soc. 41 (1973), 197-200. MR 49:5782

11. C. Finet and W. Schachermayer, Equivalent norms on separable Asplund spaces, Studia Math. 92 (1989), 275-283. MR 90d:46026

12. G. Godefroy and N. J. Kalton, Approximating sequences and bidual projections, Quart. J. Math. Oxford (2) 48 (1997), 179-202. CMP 97:15

13. G. Godefroy, N. J. Kalton, and D. Li, Propriété d'approximation métrique inconditionnelle et sous-espaces de $L^{1}$ dont la boule est compact en mesure, C. R. Acad. Sci. Paris Sér. I Math. 320 (1995), 1069-1073. MR 96d:46015

14. G. Godefroy, N. J. Kalton, and D. Li, On subspaces of $L^{1}$ which embed into $\ell_{1}$, J. Reine Angew. Math. 471 (1996), 43-75. MR 97d:46017

15. G. Godefroy, N. J. Kalton, and P. D. Saphar, Idéaux inconditionnels dans les espaces de Banach, C. R. Acad. Sci. Paris Sér. I 313 (1991), 845-849. MR 93h:46013

16. G. Godefroy, N. J. Kalton, and P. D. Saphar, Unconditional ideals in Banach spaces, Studia Math. 104 (1993), 13-59. MR 94k:46024

17. G. Godefroy and P. D. Saphar, Duality in spaces of operators and smooth norms on Banach spaces, Illinois J. Math. 32 (1988), 672-695. MR 89j:47026

18. N. Grønbæk and G. A. Willis, Approximate identities in Banach algebras of compact operators, Canad. Math. Bull. 36 (1993), 45-53. MR 94b:46076

19. P. Habala, P. Hájek, and V. Zizler, Introduction to Banach spaces, I, Charles University, Prague, 1996.

20. P. Harmand, D. Werner, and W. Werner, $M$-ideals in Banach spaces and Banach algebras, Lecture Notes in Math., vol. 1547, Springer, Berlin-Heidelberg-New York, 1993. MR 94k:46022

21. K. John, u-ideals of factorable operators, Czechoslovak. Math. J. (to appear).

22. J. Johnson, Remarks on Banach spaces of compact operators, J. Funct. Anal. 32 (1979), 304-311. MR 82k:47060

23. W. B. Johnson, Finite-dimensional Schauder decompositions in $\pi_{\lambda}$ and dual $\pi_{\lambda}$ spaces, Illinois J. Math. 14 (1970), 642-647. MR 42:826

24. W. B. Johnson, A complementary universal conjugate Banach space and its relation to the approximation problem, Israel J. Math. 13 (1972), 301-310. MR 48:4700

25. W. B. Johnson, H. P. Rosenthal, and M. Zippin, On bases, finite dimensional decompositions and weaker structures in Banach spaces, Israel J. Math. 9 (1971), 488-506. MR 43:6702

26. N. J. Kalton, Banach spaces for which the ideal of compact operators is an M-ideal, C. R. Acad. Sci. Paris Sér. I 313 (1991), 509-513. MR 93a:46027

27. N. J. Kalton, M-ideals of compact operators, Illinois J. Math. 37 (1993), 147-169. MR 94b:46028

28. N. J. Kalton and D. Werner, Property $(M), M$-ideals, and almost isometric structure of Banach spaces, J. Reine Angew. Math. 461 (1995), 137-178. MR 96m:46022

29. D. Li, Complex unconditional metric approximation property for $\mathcal{C}_{\Lambda}(\mathbf{T})$-spaces, Studia Math. 121 (1996), 231-247. MR 97k:43008

30. A. Lima, On M-ideals and best approximation, Indiana Univ. Math. J. 31 (1982), 27-36. MR 83b:46021

31. A. Lima, Property $\left(w M^{*}\right)$ and the unconditional metric compact approximation property, Studia Math. 113 (1995), 249-263. MR 96c:46019

32. J. Lindenstrauss, On nonseparable reflexive Banach spaces, Bull. Amer. Math. Soc. 72 (1966), 967-970. MR 34:4875

33. J. Lindenstrauss and L. Tzafriri, Classical Banach Spaces, I, Sequence Spaces, Springer, Berlin-Heidelberg, 1977. MR 58:17766

34. E. Oja, A note on $M$-ideals of compact operators, Tartu Ül. Toimetised 960 (1993), 75-92. MR 95a:46026 
35. E. Oja, HB-subspaces and Godun sets of subspaces in Banach spaces, Mathematika 44 (1997), 120-132. MR 98j:46008

36. E. Oja, $M$-ideals of compact operators are separably determined, Proc. Amer. Math. Soc. 126 (1998), 2747-2753. MR 98k:46032

37. E. Oja and M. Põldvere, On subspaces of Banach spaces where every functional has a unique norm-preserving extension, Studia Math. 117 (1996), 289-306. MR 97d:46016

38. R. R. Phelps, Convex Functions, Monotone Operators and Differentiability, Lecture Notes in Math., vol. 1364, Springer, Berlin-Heidelberg, 1993. MR 94f:46055

39. S. Simons, A convergence theorem with boundary, Pacific J. Math. 40 (1972), 703-708. MR 47:755

40. S. Simons, An eigenvector proof of Fatou's lemma for continuous functions, Math. Intelligencer 17 (1995), 67-70. MR 96e:26003

41. I. Singer, Bases in Banach spaces II, Editura Acad. R. S. România, Springer, Bucharest, 1981. MR 82k:46024

42. D. Werner, $M$-ideals and the "basic inequality", J. Approx. Theory 76 (1994), 21-30. MR 95i:47080

43. W. Werner, Inner M-ideals in Banach algebras, Math. Ann. 291 (1991), $205-223$. MR 93b:46094

44. G. Willis, The compact approximation property does not imply the approximation property, Studia Math. 103 (1992), 99-108. MR 93i:46035

45. M. Zippin, Banach spaces with separable duals, Trans. Amer. Math. Soc. 310 (1988), 371-379. MR 90b:46028

Faculty of Mathematics, Tartu University, Vanemuise 46, 51014 Tartu, Estonia

E-mail address: eveoja@math.ut.ee 